\title{
Asymptotic properties of type I elliptical random vectors
}

\author{
Enkelejd Hashorva
}

Received: 6 September 2006 / Revised: 31 May 2007 /

Accepted: 4 June 2007 / Published online: 24 October 2007

(C) Springer Science + Business Media, LLC 2007

\begin{abstract}
Let $\boldsymbol{X}=A \boldsymbol{S}$ be an elliptical random vector with $A \in \mathbb{R}^{k \times k}, k \geq 2$, a non-singular square matrix and $\boldsymbol{S}=\left(S_{1}, \ldots, S_{k}\right)^{\top}$ a spherical random vector in $\mathbb{R}^{k}$, and let $\boldsymbol{t}_{n}, n \geq 1$ be a sequence of vectors in $\mathbb{R}^{k}$ such that $\lim _{n \rightarrow \infty} \boldsymbol{P}\{\boldsymbol{X}>$ $\left.\boldsymbol{t}_{n}\right\}=0$. We assume in this paper that the associated random radius $R_{k}=$ $\left(S_{1}+S_{2}+\cdots+S_{k}\right)^{1 / 2}$ is almost surely positive, and it has distribution function in the Gumbel max-domain of attraction. Relying on extreme value theory we obtain an exact asymptotic expansion of the tail probability $\boldsymbol{P}\left\{\boldsymbol{X}>\boldsymbol{t}_{n}\right\}$ for $\boldsymbol{t}_{n}$ converging as $n \rightarrow \infty$ to a boundary point. Further we discuss density convergence under a suitable transformation. We apply our results to obtain an asymptotic approximation of the distribution of partial excess above a high threshold, and to derive a conditional limiting result. Further, we investigate the asymptotic behaviour of concomitants of order statistics, and the tail asymptotics of associated random radius for subvectors of $\boldsymbol{X}$.
\end{abstract}

Keywords Gumbel max-domain of attraction - Exact tail asymptotics • Density approximation - Gauss-Gumbel convergence •

Gaussian random vectors • Concomitants of order statistics •

Quadratic programming

AMS 2000 Subject Classification 60F05 • 60G70

Dedicated to Professor Samuel Kotz on the occasion of his 75th birthday.

E. Hashorva $(\bowtie)$

Allianz Suisse Insurance Company, Laupenstrasse 27,

3001 Bern, Switzerland

e-mail: enkelejd.hashorva@Allianz-Suisse.ch

E. Hashorva

Department of Mathematical Statistics and Actuarial Science,

University of Bern, Sidlerstrasse 5, 3012 Bern, Switzerland 


\section{Introduction}

Let $\boldsymbol{X}$ be an elliptical random vector in $\mathbb{R}^{k}, k \geq 2$, and let $\boldsymbol{t}_{n}, n \geq 1$ be a sequence of absorbing thresholds in $\mathbb{R}^{k}$ such that $\lim _{n \rightarrow \infty} \boldsymbol{P}\left\{\boldsymbol{X}>\boldsymbol{t}_{n}\right\}=0$. It is of theoretical interest to find a positive sequence $c_{n}, n \geq 1$ which converges to $\infty$ such that

$$
\lim _{n \rightarrow \infty} c_{n} \boldsymbol{P}\left\{\boldsymbol{X}>\boldsymbol{t}_{n}\right\}=c \in(0, \infty), \quad n \rightarrow \infty .
$$

The exact asymptotic tail behaviour of $\boldsymbol{X}$ for given thresholds $\boldsymbol{t}_{n}, n \geq 1$ is thus captured by $c$ and $c_{n}, n \geq 1$.

In this paper we use several results and ideas from extreme value theory to derive Eq. 1.1 for a large class of elliptical random vectors.

It is well-known that Gaussian random vectors belong to the larger class of elliptical random vectors (see Fang et al. 1990). Due to the central role of the Gaussian distribution in probability theory and statistics, the number of articles which have focused on Eq. 1.1 with $\boldsymbol{X}$ a standard Gaussian random vectors is huge. We mention just few recent contributions Dai and Mukherjea (2001), Hashorva and Hüsler (2002a,b, 2003), Hashorva (2003, 2005a).

To motivate our novel approach we consider briefly the specifics in the Gaussian setup from the point of view of extreme value theory.

If $\boldsymbol{X}$ is a standard Gaussian random vector in $\mathbb{R}^{k}, k \geq 1$, with non-singular covariance matrix $\Sigma$, then the following stochastic representation (see e.g. Cambanis et al. 1981; Fang et al. 1990)

$$
\boldsymbol{X} \stackrel{d}{=} R_{k} A^{\top} \boldsymbol{U}_{k}
$$

is valid with $R_{k}>0$ (almost surely) such that $R_{k}^{2}$ is chi-squared distributed with $k$ degrees of freedom, $A$ a square matrix satisfying $A^{\top} A=\Sigma$, and $\boldsymbol{U}_{k}=$ $\left(U_{1}, \ldots, U_{k}\right)^{\top}$ a random vector independent of $R_{k}$ uniformly distributed on the unit sphere of $\mathbb{R}^{k}\left(\stackrel{d}{=}\right.$ means equality of distribution functions and ${ }^{\top}$ stands for the transpose sign).

It is somewhat intuitive that the asymptotic behaviour $(n \rightarrow \infty)$ of $\boldsymbol{P}\left\{\boldsymbol{X}>\boldsymbol{t}_{n}\right\}$ is determined by the asymptotic tail behaviour of the random radius $R_{k}$. This is indeed the case as will be shown later in the paper.

From the extreme value theory we know that in the Gaussian case the random radius $R_{k}$ in Eq. 1.2 has the distribution function $F_{k}$ in the max-domain of attraction of the unit Gumbel distribution $\Lambda(x)=\exp (-\exp (-x)), x \in \mathbb{R}$. It turns out that this asymptotic behaviour of the distribution function of the associated random radius $R_{k}$ - the Gumbel max-domain of attraction-is the key to obtain the exact asymptotic tail behaviour of Gaussian random vectors.

A natural generalisation of the Gaussian case is achieved via Eq. 1.2, which leads us to elliptical random vectors.

As in the Gaussian setup, we assume throughout this paper that the random vector $\boldsymbol{X}$ in $\mathbb{R}^{k}$ has stochastic representation Eq. 1.2, where the associated 
random radius $R_{k}$ has distribution function $F_{k}$ in the Gumbel max-domain of attraction. We shall call such random vectors of Type I or alternatively Type I elliptical random vectors.

Several asymptotic results for Type I elliptical random vectors are obtained in Hashorva (2005b,c, 2006a,b,c). Motivated by the aforementioned papers which in turn are all motivated from results in the excellent monograph by Berman (1992) and the ideas in Hüsler and Reiss (1989) we derive in this paper the exact asymptotic behaviour of $\boldsymbol{P}\left\{\boldsymbol{X}>\boldsymbol{t}_{n}\right\}$ considering a general sequence of absorbing thresholds $\boldsymbol{t}_{n}, n \geq 1$ which converges to a boundary point as $n \rightarrow \infty$. Both cases $R_{k}$ is bounded and $R_{k}$ has an unbounded support are investigated. As a special case of our main result we derive the exact asymptotic behaviour for $\boldsymbol{X}$ a standard Gaussian random vector.

It is well-known (see e.g. Cambanis et al. 1981; Fang et al. 1990) that the components of elliptical random vectors possess a density function. We derive in this paper some asymptotic results for the density functions of Type I elliptical random vectors.

Motivated by asymptotic results for spherical and elliptical random vectors obtained in Berman (1992) we provide in this paper three applications for Type I elliptical random vectors; first we show an asymptotic approximation of the distribution of excesses above high thresholds. Then we discuss some conditional limiting results followed by an application concerning the asymptotic approximation of concomitants of order statistics.

The range of other applications of our results addressed in several forthcoming articles is indeed quite broad. Few such instances concerning Type I elliptical distributions are the identification of parameters of the sample minimum, asymptotics of convex hulls and related characteristics, and statistical estimation of rare events.

Due to the multivariate setup addressed in this paper the access to some results and their proofs is to not easy. After having established all the results of this paper, we have therefore addressed in a separate forthcoming paper the bivariate setup which can be dealt with more straightforwardly (see Hashorva 2007c).

In the approximations and our applications the multivariate Gaussian distribution shows up in the limit. Professor Samuel Kotz (personal communications) remarks: "The results are astonishing! Gumbel max-domain of attraction and the Gaussian distribution!! What a connection."

We coin the corresponding asymptotic results the Gauss-Gumbel convergence.

Apart from some theoretical interest, we believe that the applications presented in this paper will have a certain impact in statistical modelling of multivariate excess distributions. Several techniques and ideas are already available in the literature, see for instance Heffernan and Tawn (2004), Abdous et al. (2005), Butler and Tawn (2007), Heffernan and Resnick (2005), Abdous et al. (2006), Klüppelberg et al (2007).

We organise the paper as follows: In the next section we introduce some notation and provide a basic result for elliptical random vectors. In Section 3 
we derive an exact asymptotic expansions (as in Eq. 1.1) for Type I elliptical random vectors followed by asymptotic results in Section 4 on density convergence. Applications are provided in Section 5 followed by three illustrating examples. In Section 7 we give several other results and provide the proofs of all the results given in the previous sections. We conclude the paper with an Appendix.

\section{Preliminaries}

We shall introduce first some standard notation. Let in the following $I, J$ be two non-empty disjoint index sets such that $I \cup J=\{1, \ldots, k\}, k \geq 2$, and define for $\boldsymbol{x}=\left(x_{1}, \ldots, x_{k}\right)^{\top} \in \mathbb{R}^{k}$ the subvector with respect to the index set $I$ by $\boldsymbol{x}_{I}:=\left(x_{i}, i \in I\right)^{\top} \in \mathbb{R}^{k}$. If $\Sigma \in \mathbb{R}^{k \times k}$ is a square matrix then the matrix $\Sigma_{I J}$ is obtained by deleting both the rows and the columns of $\Sigma$ with indices in $J$ and in $I$, respectively. Similarly we define $\Sigma_{J I}, \Sigma_{J J}, \Sigma_{I I}$. For notational simplicity we write $\boldsymbol{x}_{I}^{\top}, \Sigma_{J J}^{-1}$ instead of $\left(\boldsymbol{x}_{I}\right)^{\top},\left(\Sigma_{J J}\right)^{-1}$, respectively, and set $\boldsymbol{x}_{u, I}$ instead of $\left(\boldsymbol{x}_{u}\right)_{I}$ if $\boldsymbol{x}_{u}$ is a vector indexed by $u$. We shall define (given $\boldsymbol{a}, \boldsymbol{x}, \boldsymbol{y} \in \mathbb{R}^{k}$ )

$$
\begin{aligned}
\boldsymbol{x} & >\boldsymbol{y}, \text { if } x_{i}>y_{i}, \quad \forall i=1, \ldots, k, \\
\boldsymbol{x} & \geq \boldsymbol{y}, \text { if } x_{i} \geq y_{i}, \quad \forall i=1, \ldots, k, \\
\boldsymbol{x}+\boldsymbol{y} & :=\left(x_{1}+y_{1}, \ldots, x_{k}+y_{k}\right)^{\top}, \\
c \boldsymbol{x} & :=\left(c x_{1}, \ldots, c x_{k}\right)^{\top}, \quad c \in \mathbb{R}, \\
\boldsymbol{a x} & :=\left(a_{1} x_{1}, \ldots, a_{k} x_{k}\right)^{\top}, \quad \boldsymbol{x} / \boldsymbol{a}:=\left(x_{1} / a_{1}, \ldots, x_{k} / a_{k}\right)^{\top}, \\
\mathbf{0} & :=(0, \ldots, 0)^{\top} \in \mathbb{R}^{k}, \quad \mathbf{1}:=(1, \ldots, 1)^{\top} \in \mathbb{R}^{k} . \\
\left\|\boldsymbol{x}_{I}\right\|^{2} & :=\boldsymbol{x}_{I}^{\top} \Sigma_{I I}^{-1} \boldsymbol{x}_{I}, \quad \mathcal{S}^{m-1}:=\left\{\boldsymbol{x} \in \mathbb{R}^{m}: \boldsymbol{x}^{\top} \boldsymbol{x}=1\right\}, \quad m \geq 1 .
\end{aligned}
$$

If a random vector $\boldsymbol{Y}$ possesses the distribution function $H$, we shall indicate this by $\boldsymbol{Y} \sim H$. We shall be denoting by $\mathcal{B}_{a, b}$ a Beta random variable with parameters $a$ and $b$ and density function

$$
\frac{\Gamma(a+b)}{\Gamma(a) \Gamma(b)} x^{a-1}(1-x)^{b-1}, \quad x \in(0,1)
$$

with $\Gamma(\cdot)$ the Gamma function. $\mathcal{E}_{\mu}$ denotes an Exponential random variable with mean $\mu \in(0, \infty)$.

Let $\boldsymbol{S}:=\left(S_{1}, \ldots, S_{k}\right)^{\top}$ be a spherical random vector in $\mathbb{R}^{k}, k \geq 2$. The random vector $\boldsymbol{S}$ is spherically distributed, if $\boldsymbol{S} \stackrel{d}{=} O \boldsymbol{S}$ holds for any orthogonal matrix $O \in \mathbb{R}^{k \times k}$. See e.g. Cambanis et al. (1981); Fang et al. (1990) or Berman (1992). The next lemma provides a general result on the linear combinations of spherical random vectors. 
Lemma 2.1 [Lemma 6.1, Berman (1983), Lemma 12.1.2, Berman (1992)]. Let $\boldsymbol{S}=\left(S_{1}, \ldots, S_{k}\right)^{\top}, k \geq 2$, be a spherical random vector in $\in \mathbb{R}^{k}$ and let $\boldsymbol{a}_{1}, \ldots, \boldsymbol{a}_{i}, i \leq k$, be given vectors in $\mathbb{R}^{k}$ such that $\boldsymbol{a}_{i}^{\top} \boldsymbol{a}_{k}=0,1 \leq l<j \leq k$. Then we have

$$
\left(\boldsymbol{a}_{1}^{\top} \boldsymbol{S}, \ldots, \boldsymbol{a}_{i}^{\top} \boldsymbol{S}\right) \stackrel{d}{=}\left(\left(\boldsymbol{a}_{1}^{\top} \boldsymbol{a}_{1}\right)^{1 / 2} S_{1}, \ldots,\left(\boldsymbol{a}_{1}^{\top} \boldsymbol{a}_{i}\right)^{1 / 2} S_{i}\right)
$$

Throughout this paper $\boldsymbol{U}_{m}:=\left(U_{1}, \ldots, U_{m}\right)^{\top}, m \geq 2$, stands for a uniformly distributed random vector on $\mathcal{S}^{m-1}$. Cambanis et al. (1981) show that if the associated random radius $R_{k}:=\left(\sum_{i=1}^{k} S_{i}^{2}\right)^{1 / 2}$ is almost surely positive, then the stochastic representation $\boldsymbol{S} \stackrel{d}{=} R_{k} \boldsymbol{U}_{k}$ is valid with $R_{k}$ independent of $\boldsymbol{U}_{k}$.

Elliptical random vectors are obtained by linear transformations of the spherical ones. The basic distributional properties of elliptical random vectors can be found in Kotz (1975), Cambanis et al. (1981), Fang et al. (1990), Anderson and Fang (1990), Szabłowski (1990), Fang and Zhang (1990), Berman (1992), Gupta and Varga (1993), Kano (1994) and Kotz and Ostrovskii (1994), among many others.

In this paper we consider an elliptical random vector $\boldsymbol{X}=\left(X_{1}, \ldots, X_{k}\right)^{\top}$ with stochastic representation

$$
\boldsymbol{X} \stackrel{d}{=} A^{\top} \stackrel{S}{=} \stackrel{d}{=} R_{k} A^{\top} \boldsymbol{U}_{k},
$$

where $A \in \mathbb{R}^{k \times k}$ is a non-singular matrix. $\Sigma:=A^{\top} A$ is a positive definite matrix with positive determinant $|\Sigma|$. Without loss of generality we assume in the sequel that $\Sigma$ is a correlation matrix, i.e. all the entries of the main diagonal of $\Sigma$ are equal 1. Lemma 2.1 implies then $X_{i} \stackrel{d}{=} S_{1}, 1 \leq i \leq k$.

As in the Gaussian case (see Hashorva 2005a) for the tail asymptotic expansion of interest the solution of the quadratic programming problem

$$
\mathcal{P}\left(\Sigma^{-1}, \boldsymbol{t}_{n}\right) \text { : minimise }\|\boldsymbol{x}\|^{2}=\boldsymbol{x}^{\top} \Sigma^{-1} \boldsymbol{x} \text { under the linear constraint } \boldsymbol{x} \geq \boldsymbol{t}_{n},
$$

with $\boldsymbol{t}_{n}$ a threshold in $\mathbb{R}^{k}$ is crucial. If the Savage condition (see Hashorva 2005a for more details)

$$
\Sigma^{-1} \boldsymbol{t}_{n}>\mathbf{0}
$$

is satisfied, then the minimum is attained at $\boldsymbol{t}_{n}$, otherwise there exists a unique index set $I \subset\{1, \ldots, k\}$ which defines the unique solution of $\mathcal{P}\left(\Sigma^{-1}, \boldsymbol{t}_{n}\right)$ (see Proposition 7.1 below). We will refer to the aforementioned index set $I$ in the following simply as the minimal index set.

\section{Tail Asymptotics}

Let $\boldsymbol{X}$ be an elliptical random vector in $\mathbb{R}^{k}, k \geq 2$, with stochastic representation (Eq. 2.4), and set $\Sigma:=A^{\top} A$ with $A$ a non-singular $k$-dimensional square matrix. In the rest of the paper we assume that $\boldsymbol{X}$ is of Type I, i.e. the distribution function $F_{k}$ of the random radius $R_{k}$ is in the Gumbel 
max-domain of attraction. This means that there exists a positive scaling function $w$ such that

$$
\lim _{u \uparrow \omega} \frac{1-F_{k}(u+x / w(u))}{1-F_{k}(u)}=\exp (-x), \quad \forall x \in \mathbb{R},
$$

with $\omega:=\sup \left\{s: F_{k}(s)<1\right\}$ the upper endpoint of the distribution function $F_{k}$. We shall denote the above asymptotic relation by $F_{k} \in M D A(\Lambda, w)$, and refer the reader for a deeper insight in the extreme value theory to the following standard monographs: De Haan (1970), Leadbetter et al. (1983), Galambos (1987), Resnick (1987), Reiss (1989), Kotz and Nadarajah (2000), Falk et al. (2004), or De Haan and Ferreira (2006).

For Type I elliptical random vectors we shall obtain in this section an exact asymptotic expansion of $\boldsymbol{P}\left\{\boldsymbol{X}>\boldsymbol{t}_{n}\right\}$ as $n \rightarrow \infty$, with $\boldsymbol{t}_{n}, n \geq 1$ a given sequence of absorbing thresholds tending to a boundary point. If $\omega$ is finite (we assume without loss of generality $\omega=1$ ) in order to avoid trivial thresholds we suppose additionally that $0<\left\|\boldsymbol{t}_{n}\right\|<\omega, \forall n \geq 1$.

In the last part of this section we discuss the sensitivity of our asymptotic expansion by investigating the asymptotics of the ratio $\boldsymbol{P}\left\{\boldsymbol{X}>\boldsymbol{t}_{n}\right\} / \boldsymbol{P}\{\boldsymbol{X}>$ $\left.\boldsymbol{t}_{n}^{*}\right\}, n \rightarrow \infty$, with $\boldsymbol{t}_{n}^{*}, n \geq 1$ another sequence of thresholds.

In the sequel $\boldsymbol{Z}$ stands for a Gaussian random vector in $\mathbb{R}^{k}$ with covariance matrix $\Sigma$, and set

$$
\alpha_{n}:=\left\|\boldsymbol{t}_{n, I}\right\|, \quad \beta_{n}:=w\left(\alpha_{n}\right), \quad n \geq 1,
$$

with $I \subset\{1, \ldots, k\}$ a non-empty index set. We consider first the case $\omega$ is infinite.

Case $\omega=\infty$ Clearly, if the constants $c_{n}, n \geq 1$ in Eq. 1.1 are positive and tend to infinity as $n \rightarrow \infty$, then the sequence of threshold $\boldsymbol{t}_{n}$ necessarily satisfies $\lim _{n \rightarrow \infty}\left\|\boldsymbol{t}_{n}\right\|=\infty$. In the Gaussian case we know (relying for instance on the large deviation theory) that the solution of quadratic programming problem $\mathcal{P}\left(\Sigma^{-1}, \boldsymbol{t}_{n}\right), n \geq 1$ is crucial for determining the asymptotic behaviour of $\boldsymbol{P}\left\{\boldsymbol{X}>\boldsymbol{t}_{n}\right\}, n \rightarrow \infty$. As shown in the main theorem below the same holds true for the more general setup of elliptical random vectors of Type I.

With slight abuse of notation we denote in the following by $\boldsymbol{e}_{i}$ (and not $\boldsymbol{e}_{i, I}$ ) the $i$-th unit vector in $\mathbb{R}^{|I|}$, where $|I| \geq 1$ is the number of elements of the index set $I$.

Theorem 3.1 Let $\boldsymbol{X} \stackrel{d}{=} R_{k} A^{\top} \boldsymbol{U}_{k}$ be an elliptical random vector in $\mathbb{R}^{k}, k \geq 2$, with $R_{k}$ an almost surely positive random radius with distribution function $F_{k}$ independent of $\boldsymbol{U}_{k}$ and $A \in \mathbb{R} R^{k \times k}$ a non-singular matrix. Suppose that $F_{k}$ satisfies Eq. 3.7 with $\omega=\infty$ and the positive scaling function $w$. Let $\boldsymbol{t}, \boldsymbol{t}_{n}, n \geq 1$ be thresholds in $\mathbb{R}^{k} \backslash(-\infty, 0]^{k}$, and for $n \in \mathbb{N}$ let $I_{n}$ be the minimal index set corresponding to the quadratic programming problem $\mathcal{P}\left(\Sigma^{-1}, \boldsymbol{t}_{n}\right)$ with $\Sigma:=$ $A^{\top}$ A. Assume that

$$
I_{n}=I, \quad \forall n \geq 1, \quad \boldsymbol{t}_{I}^{\top} \Sigma_{I I}^{-1} \boldsymbol{e}_{i}>0, \quad \forall i \in I,
$$


and

$$
\lim _{n \rightarrow \infty} \alpha_{n}=\omega, \quad \lim _{n \rightarrow \infty} \frac{\boldsymbol{t}_{n, I}}{\alpha_{n}}=\boldsymbol{t}_{I}
$$

holds with $\alpha_{n}, \beta_{n}$ as in Eq. 3.8.

If further

$$
\lim _{n \rightarrow \infty}\left(\frac{\beta_{n}}{\alpha_{n}}\right)^{1 / 2}\left(\boldsymbol{t}_{n, J}-\Sigma_{J I} \Sigma_{I I}^{-1} \boldsymbol{t}_{n, I}\right)=\widetilde{\boldsymbol{u}}_{J} \in[-\infty, 0]^{|J|},
$$

is satisfied in the case $1 \leq|I|<k$, then we have the asymptotic expansion $(n \rightarrow \infty)$

$$
\begin{aligned}
\boldsymbol{P}\left\{\boldsymbol{X}>\boldsymbol{t}_{n}\right\}= & (1+o(1)) \times \frac{\Gamma(k / 2) 2^{k / 2-1} \boldsymbol{P}\left\{\boldsymbol{Z}_{J}>\tilde{\boldsymbol{u}}_{J} \mid \boldsymbol{Z}_{I}=\mathbf{0}_{I}\right\}}{(2 \pi)^{|I| / 2}\left|\Sigma_{I I}\right|^{1 / 2} \prod_{i \in I} \boldsymbol{t}_{I}^{\top} \Sigma_{I I}^{-1} \boldsymbol{e}_{i}} \\
& \times\left(\alpha_{n} \beta_{n}\right)^{1+|J| / 2-k}\left(1-F_{k}\left(\alpha_{n}\right)\right)
\end{aligned}
$$

Set $\boldsymbol{P}\left\{\boldsymbol{Z}_{J}>\widetilde{\boldsymbol{u}}_{J} \mid \boldsymbol{Z}_{I}=\mathbf{0}_{I}\right\}$ to 1 if $|I|=k$.

\section{Remark 3.2}

(a) Under the assumptions of Theorem 3.1 we obtain using further Eq. 3.16

$$
\lim _{n \rightarrow \infty} \alpha_{n} \beta_{n}=\infty
$$

Consequently $\boldsymbol{P}\left\{\boldsymbol{X}>\boldsymbol{t}_{n}\right\}$ decreases faster than $1-F\left(\alpha_{n}\right)$ as $n \rightarrow \infty$.

(b) The scaling function $w$ in Eq. 3.7 can be defined asymptotically by

$$
w(u):=\frac{(1+o(1))[1-F(u)]}{\int_{u}^{\omega}[1-F(s)] d s}, \quad u \uparrow \omega,
$$

and uniformly for $x$ in compact sets of $\mathbb{R}$

$$
\lim _{u \uparrow \omega} \frac{w(u+x / w(u))}{w(u)}=1 .
$$

Furthermore, setting $k(u):=u$ if $\omega=\infty$, and $k(u):=\omega-u$ otherwise we have

$$
\lim _{u \uparrow \omega} k(u) w(u)=\infty .
$$

Case $\omega=1$ We deal next with the case $F_{k}$ has a finite upper endpoint, assuming for simplicity $\omega=1$. The distribution function of $\boldsymbol{X}$ has the support on the ellipsoid defined by $\Sigma^{-1}$ and $\omega . \boldsymbol{P}\left\{\boldsymbol{X}>\boldsymbol{t}_{n}\right\}$ does not seems very interesting quantity in this case, since depending on the threshold $\boldsymbol{t}_{n}$, the random vector $\boldsymbol{X}$ might not put mass at all on the set $D_{n}:=\left\{\boldsymbol{x} \in \mathbb{R}^{k}: \boldsymbol{x}>\boldsymbol{t}_{n}\right\}$. We deal however only with $\boldsymbol{P}\left\{\boldsymbol{X}>\boldsymbol{t}_{n}\right\}$ since the idea can be carried out to the general case $\boldsymbol{P}\left\{\boldsymbol{X} \in C_{n}\right\}$ with $C_{n} \subset \mathbb{R}^{k}, n \geq 1$ a sequence of absorbing Borel sets satisfying $\lim _{n \rightarrow \infty} \boldsymbol{P}\left\{\boldsymbol{X} \in C_{n}\right\}=0$. The special case $C_{n}$ is defined in terms of linear transformations of $D_{n}$ is very tractable (recall Lemma 2.1).

In order to consider absorbing events the sequence of thresholds $t_{n}, n \geq 1$, necessarily should converge to a point $t$ such that $\|\boldsymbol{t}\| \geq \omega$. If $\Sigma=A^{\top} A$ is the 
identity matrix and $\boldsymbol{t}$ has at least one negative component, then $\boldsymbol{P}\{\boldsymbol{X} \geq \boldsymbol{t}\}$ is positive. Thus we need to impose some restrictions on $\boldsymbol{t}$ which yield $\boldsymbol{P}\{\boldsymbol{X}>\boldsymbol{t}\}=0$.

Theorem 3.3 Let $A, \boldsymbol{e}_{i}, F_{k}, R_{k}, \boldsymbol{U}_{k}, \boldsymbol{X}, \boldsymbol{Z}, \Sigma$ be as in Theorem 3.1, and let further $\boldsymbol{t}, \boldsymbol{t}_{n}, n \geq 1$ be a sequence of thresholds in $\mathbb{I R}^{k} \backslash(-\infty, 0]^{k}$ such that $0<\left\|\boldsymbol{t}_{n}\right\|<1, \forall n \geq 1$. Assume that $F_{k}$ satisfies Eq. 3.7 with the upper endpoint $\omega=1$ and the positive scaling function $w$. If Eqs. 3.9, 3.10 and 3.11 (if $|I|<k$ ) hold with $\alpha_{n}:=\left\|\boldsymbol{t}_{n, I}\right\|, \beta_{n}:=w\left(\alpha_{n}\right), n \geq 1$, then we have

$$
\begin{aligned}
\boldsymbol{P}\left\{\boldsymbol{X}>\boldsymbol{t}_{n}\right\}=(1+o(1)) & \frac{\Gamma(k / 2) 2^{k / 2-1} \boldsymbol{P}\left\{\boldsymbol{Z}_{J}>\widetilde{\boldsymbol{u}}_{J} \mid \boldsymbol{Z}_{I}=\mathbf{0}_{I}\right\}}{(2 \pi)^{|I| / 2}\left|\Sigma_{I I}\right|^{1 / 2} \prod_{i \in I} \boldsymbol{t}_{I}^{\top} \Sigma_{I I}^{-1} \boldsymbol{e}_{i}} \beta_{n}^{1+|J| / 2-k} \\
& \times\left(1-F_{k}\left(\alpha_{n}\right)\right), \quad n \rightarrow \infty
\end{aligned}
$$

If $|I|=k$ set $\boldsymbol{P}\left\{\boldsymbol{Z}_{J}>\widetilde{\boldsymbol{u}}_{J} \mid \boldsymbol{Z}_{I}=\mathbf{0}_{I}\right\}$ to 1 .

It is of some interest to know how do $c_{n}, n \geq 1$ and $c$ in Eq. 1.1 change if we consider a new sequence of thresholds $\boldsymbol{t}_{n}^{*}:=\boldsymbol{t}_{n}+\boldsymbol{q}_{n}, n \geq 1$ instead of $\boldsymbol{t}_{n}$ with $\boldsymbol{q}_{n} \in \mathbb{R}^{k}, n \geq 1$ another perturbating sequence. We shall show in the next theorem that under an asymptotic condition on $\boldsymbol{q}_{n}$ (see below Eq. 3.19) the asymptotic behaviour of $\boldsymbol{P}\left\{\boldsymbol{X}>\boldsymbol{t}_{n}^{*}\right\}$ is the same (up to a constant) as the asymptotic behaviour of $\boldsymbol{P}\left\{\boldsymbol{X}>\boldsymbol{t}_{n}\right\}, n \rightarrow \infty$.

Theorem 3.4 Let $\boldsymbol{X} \stackrel{d}{=} R_{k} A^{\top} \boldsymbol{U}_{k}$ be an elliptical random vector as in Theorem 3.1 , and let $\boldsymbol{t}, \boldsymbol{t}_{n}, n \geq 1$ be thresholds in $\mathbb{R}^{k} \backslash(-\infty, 0]^{k}$. Assume that the distribution function $F_{k}$ of $R_{k}$ has upper endpoint $\omega \in(0, \infty]$ and $F_{k} \in \operatorname{MD} A(\Lambda, w)$. Define $\boldsymbol{v}_{n}, n \geq 1$ a sequence of vectors in $\mathbb{R}^{k}$ by

$$
\boldsymbol{v}_{n, I}:=\beta_{n} \mathbf{1}_{I}, \quad \boldsymbol{v}_{n, J}:=\left(\frac{\beta_{n}}{\alpha_{n}}\right)^{1 / 2} \mathbf{1}_{J}, \quad \forall n \geq 1,
$$

with $\alpha_{n}, \beta_{n}, n \geq 1$ as in Eq. 3.8. Let $\boldsymbol{q}_{n}, n \geq 1$ be vectors in $\mathbb{R}^{k}$ such that

$$
\lim _{n \rightarrow \infty} \boldsymbol{v}_{n} \boldsymbol{q}_{n}=\boldsymbol{q}
$$

with $\boldsymbol{q}_{I} \in \mathbb{R}^{|I|}, \boldsymbol{q}_{J} \in[-\infty, \infty)^{|J|}$. If Eqs. 3.9, 3.10 and 3.11 (if $|I|<k$ ) hold, then we have

$$
\lim _{n \rightarrow \infty} \frac{\boldsymbol{P}\left\{\boldsymbol{X}>\boldsymbol{t}_{n}+\boldsymbol{q}_{n}\right\}}{\boldsymbol{P}\left\{\boldsymbol{X}>\boldsymbol{t}_{n}\right\}}=\exp \left(-\boldsymbol{t}_{I}^{\top} \Sigma_{I I}^{-1} \boldsymbol{q}_{I}\right) \frac{\boldsymbol{P}\left\{\boldsymbol{Z}_{J}>\widetilde{\boldsymbol{u}}_{J}+\boldsymbol{q}_{J} \mid \boldsymbol{Z}_{I}=\mathbf{0}_{I}\right\}}{\boldsymbol{P}\left\{\boldsymbol{Z}_{J}>\widetilde{\boldsymbol{u}}_{J} \mid \boldsymbol{Z}_{I}=\mathbf{0}_{I}\right\}} .
$$

In the next lemma we show that our results can be stated for a subvector $\boldsymbol{X}_{K}, K \subset\{1, \ldots, k\}$ of $\boldsymbol{X}$.

钐 Springer 
Lemma 3.5 Let $\boldsymbol{X}=R_{k} \boldsymbol{U}_{k}$ be a Type I elliptical random vector with $F_{k}$ the distribution function of $R_{k}$ satisfying Eq. 3.7. Then for any non-empty subset $K$ of $\{1, \ldots, k\}$ we have $\boldsymbol{X}_{K} \stackrel{d}{=} R_{m} \boldsymbol{U}_{m}, m:=|K| \geq 1$ where $R_{m}>0$ has distribution function $F_{m}$ in the Gumbel max-domain of attraction with the scaling function w. Furthermore, $R_{m}$ is independent of $\boldsymbol{U}_{m}$.

We note that in view of Theorem 12.3.1 and Lemma 12.1.2 of Berman (1992) (see also Hashorva 2005b, 2007a) the components of the random vector $\boldsymbol{X}$ in Theorem 3.1 have distribution functions in the Gumbel max-domain of attraction with the scaling function $w$. This fact is well-known for $\boldsymbol{X}$ a standard Gaussian random vector, see e.g. Leadbetter et al. (1983) or Resnick (1987).

\section{Density Approximation}

Elliptical random vectors are very tractable due to Eq. 1.2 which describes the distribution function of such vectors in terms of the distribution function of the associated random radius $R_{k}$ and the uniform distribution on the unit sphere. For a subclass of elliptical random vectors it is possible to obtain an explicit formula for their density function. Explicitly, let $\boldsymbol{X}$ be a $k$-dimensional random vector with stochastic representation (Eq. 1.2) where $R_{k}>0$ has distribution function $F_{k}$ and $A$ is a non-singular $k \times k$ real matrix. Define the class $\mathcal{M}_{R_{k}}$ of positive random variables by

$$
\mathcal{M}_{R_{k}}:=\left\{R_{n}>0, n \in \mathbb{N}, n \geq k: R_{k}^{2} \stackrel{d}{=} R_{n}^{2} \mathcal{B}_{k / 2,(n-k) / 2}\right\},
$$

(recall $\mathcal{B}_{a, b}$ in our notation denotes a Beta distributed random variable with positive parameters $a, b)$. If $n=k$ we set $\mathcal{B}_{k / 2,0}:=1$ implying thus $R_{k} \in \mathcal{M}_{R_{k}}$. We shall define the $i$-th random radius associated to the random vector $\boldsymbol{X}$ by

$$
R_{i}:=R_{k}\left(\sum_{i=1}^{i} U_{i}^{2}\right)^{1 / 2}, \quad 1 \leq i<k,
$$

where $R_{k}$ is independent of $\boldsymbol{U}_{k}=\left(U_{1}, \ldots, U_{k}\right)^{\top}$. As shown in Cambanis et al. (1981) we have the stochastic representation

$$
R_{i}^{2} \stackrel{d}{=} R_{k}^{2} \mathcal{B}_{i / 2,(k-i) / 2}, \quad 1 \leq i<k
$$

implying $\mathcal{M}_{R_{j}} \subset \mathcal{M}_{R_{i}}, 1 \leq i<j \leq k$.

In the following we assume that $\mathcal{M}_{R_{k}}$ has at least two elements, say $R_{k}$ and $R_{d}$ with $d>k$. This implies that $\boldsymbol{X}$ possesses density function $f$ (see Lemma 7.9 below) given by

$$
f(\boldsymbol{x})=\frac{\Gamma(d / 2)}{\Gamma((d-k) / 2) \pi^{k / 2}|\Sigma|^{1 / 2}} \int_{\|\boldsymbol{x}\|}^{\infty}\left(r^{2}-\|\boldsymbol{x}\|^{2}\right)^{(d-k) / 2-1} r^{-(d-2)} d F_{d}(r), \quad \forall \boldsymbol{x} \in \mathbb{R}^{k},
$$

with $F_{d}$ the distribution function of $R_{d}$ with the upper endpoint $\omega \in(0, \infty]$, and $\Sigma:=A^{\top} A$. 
Let $\boldsymbol{t}_{n}, n \geq 1$, be a given sequence of thresholds in $\mathbb{R}^{k}$ such that $\lim _{n \rightarrow \infty}\left\|\boldsymbol{t}_{n}\right\|=\omega$. If $f$ is the density of a standard Gaussian random vector in $\mathbb{R}^{k}$ it is easy to show an asymptotic approximation of $f$ along $\boldsymbol{t}_{n}$ as follows:

$$
\lim _{n \rightarrow \infty} a_{n} f\left(\boldsymbol{t}_{n}+\boldsymbol{x} / \boldsymbol{v}_{n}\right)=\psi(\boldsymbol{x}), \quad \forall \boldsymbol{x} \in \mathbb{R}^{k}
$$

holds with $\boldsymbol{v}_{n}, n \geq 1$ another sequence of vectors in $\mathbb{R}^{k}, a_{n}, n>1$ a positive sequence of normalising constants converging to 0 , and $\psi$ a positive function in $\mathbb{R}^{k}$.

We show that Type I elliptical random vectors behave like Gaussian random vectors also in terms of density convergence. The normalising constant $a_{n}$ and the vector $\boldsymbol{v}_{n}$ depend on the asymptotic behaviour of $F_{k}$, whereas the limiting function $\psi$ is the same as in the Gaussian case. Application of the next theorems will be presented in Section 5.

Theorem 4.1 Let $A, k, F_{k}, R_{k}, \boldsymbol{X}, \omega$ be as in Theorem 3.1. Assume that $\omega \in$ $(0, \infty]$ and $F_{k} \in M D A(\Lambda, w)$ holds, and let $\boldsymbol{t}_{n}, n \geq 1$ be a sequence of vectors in $\mathbb{I R}^{k}$ such that $\lim _{n \rightarrow \infty}\left\|\boldsymbol{t}_{n}\right\|=\omega$. If $\mathcal{M}_{R_{k}}$ has at least two elements, then $\boldsymbol{X}$ possesses a density function $f$ given in Eq. 4.22 with $R_{d} \in \mathcal{M}_{R_{k}}, d>k$ such that

$$
\lim _{n \rightarrow \infty} \frac{f\left(\boldsymbol{t}_{n}\right)}{\left\|\boldsymbol{t}_{n}\right\|^{1-k} w\left(\left\|\boldsymbol{t}_{n}\right\|\right)\left[1-F_{k}\left(\left\|\boldsymbol{t}_{n}\right\|\right)\right]}=\frac{\Gamma(k / 2)}{2 \pi^{k / 2}|\Sigma|^{1 / 2}} .
$$

Utilising the above asymptotic result we show that Eq. 4.23 holds for special thresholds.

Corollary 4.2 Under the assumptions of Theorem 4.1, if we assume further that for a given non-empty index set $I \subset\{1, \ldots, k\}$ we have $\lim _{n \rightarrow \infty}\left\|\boldsymbol{t}_{n, I}\right\|=\omega$, then $\boldsymbol{X}$ possesses a density function $f$ given in Eq. 4.22 with $R_{d} \in \mathcal{M}_{R_{k}}, d>k$ such that for any $\boldsymbol{y} \in \mathbb{R}^{k}$ we have

$$
\lim _{n \rightarrow \infty} \frac{f\left(\boldsymbol{t}_{n}^{*}+\boldsymbol{y} / \boldsymbol{v}_{n}\right)}{\alpha_{n}^{1-k} w\left(\left\|\boldsymbol{t}_{n}^{*}+\boldsymbol{y} / \boldsymbol{v}_{n}\right\|\right)\left[1-F_{k}\left(\left\|\boldsymbol{t}_{n}^{*}+\boldsymbol{y} / \boldsymbol{v}_{n}\right\|\right)\right]}=\frac{\Gamma(k / 2)}{2 \pi^{k / 2}|\Sigma|^{1 / 2}},
$$

with $\boldsymbol{t}_{n, I}^{*}:=\boldsymbol{t}_{n, I}, \boldsymbol{t}_{n, J}^{*}:=\Sigma_{J I} \Sigma_{I I}^{-1} \boldsymbol{t}_{n, I}$ and $\alpha_{n}, \beta_{n}, \boldsymbol{v}_{n}, n \geq 1$ defined in Eqs. 3.8, 3.18. If $\lim _{n \rightarrow \infty} \boldsymbol{t}_{n, I} / \alpha_{n}=\boldsymbol{t}_{I}$, with $\boldsymbol{t}_{I} \in \mathbb{R}^{|I|}$ or $\boldsymbol{y} \in \mathbb{R}^{k}: \boldsymbol{y}_{I}=\mathbf{0}_{I}$, then

$$
\begin{aligned}
& \frac{f\left(\boldsymbol{t}_{n}^{*}+\boldsymbol{y} / \boldsymbol{v}_{n}\right)}{\alpha_{n}^{1-k} \beta_{n}\left[1-F_{k}\left(\alpha_{n}\right)\right]} \rightarrow \frac{(1+o(1)) \Gamma(k / 2)}{2 \pi^{k / 2}|\Sigma|^{1 / 2}} \\
& \quad \times \exp \left(-\boldsymbol{y}_{J}^{\top}\left(\Sigma^{-1}\right)_{J J} \boldsymbol{y}_{J} / 2-\boldsymbol{t}_{I}^{\top} \Sigma_{I I}^{-1} \boldsymbol{y}_{I}\right), \quad n \rightarrow \infty
\end{aligned}
$$

holds locally uniformly in $\mathbb{R}^{k}$, or in $\mathbb{R}^{|J|}$, respectively. If $|J|=0$, then $\boldsymbol{v}_{n}=$ $\beta_{n} \mathbf{1}, n \geq 1$, and put in Eq. 4.260 instead of $\boldsymbol{y}_{J}^{\top}\left(\Sigma^{-1}\right)_{J J} \boldsymbol{y}_{J}$.

\section{Remark 4.3}

(a) The results for the density convergence above are shown under the assumption $\mathcal{M}_{R_{k}}$ has at least two elements. If this is not the case, the above asymptotic results hold for the density function of $\boldsymbol{X}_{K}$ with $K$ a 
non-empty index set with $m<k$ elements, since by Lemma $3.5 \boldsymbol{X}_{K}$ is again a Type I elliptical random vector.

(b) We do not make any explicit assumption on the density function of $\boldsymbol{X}$. If the marginal distributions of $\boldsymbol{X}$ possess a differentiable density function, then the condition $\mathcal{M}_{R_{k}}$ has at least two elements is fulfilled, see Fang et al. (1990) p.37.

\section{Applications}

In the recent paper Hashorva (2006a) conditional limiting results are derived for Type I elliptical random vectors. Those results actually were motivated by Berman (1992) and indirectly from the proof of the main theorem in Hüsler and Reiss (1989). In this section we consider three applications.

Explicitly, let $\boldsymbol{X}$ be a $k$-dimensional random vector, and let $\boldsymbol{t}_{n}, n \geq 1$, be vectors in $\mathbb{R}^{k}, k \geq 1$. Define a new sequence of random vectors by the stochastic representation

$$
\boldsymbol{X}_{\left[\boldsymbol{t}_{n} ; K\right]} \stackrel{d}{=} \boldsymbol{X}-\boldsymbol{t}_{n} \mid \boldsymbol{X}_{K}>\boldsymbol{t}_{n, K}, \quad n \geq 1,
$$

where $K$ is a non-empty subset of $\{1, \ldots, k\}$. If $K=\{1, \ldots, k\}$ we write simply $\boldsymbol{X}_{\left[\boldsymbol{t}_{n}\right]}$ instead of $\boldsymbol{X}_{\left[\boldsymbol{t}_{n} ; K\right]}$. We shall call $\boldsymbol{X}_{\left[\boldsymbol{t}_{n} ; K\right]}$ the (partial) excess of $\boldsymbol{X}$ with respect to the threshold $\boldsymbol{t}_{n}$ and the index set $K$.

Derivation of the asymptotic behaviour of $\boldsymbol{X}_{\left[\boldsymbol{t}_{n} ; K\right]}$ for Type I elliptical random vectors opens the way for application of the POT method (see e.g. Falk et al. 2004) to this large class of multivariate random vectors.

As shown in Hashorva (2006a) the asymptotic behaviour of $\boldsymbol{X}_{\left[\boldsymbol{t}_{n} ;\right]}$ is related to the asymptotic behaviour of the conditional random sequence $\boldsymbol{X}_{J} \mid \boldsymbol{X}_{I}=\boldsymbol{t}_{n, I}$. In the aforementioned paper a Gaussian approximation of $\boldsymbol{X}_{J} \mid \boldsymbol{X}_{I}=\boldsymbol{t}_{n, I}$ is obtained letting $\boldsymbol{t}_{n, I}$ tend to a boundary point.

In our second application we obtain a finer result by approximating the density function of $\boldsymbol{X}_{J} \mid \boldsymbol{X}_{I}=\boldsymbol{t}_{n, I}$. Our last application concerns the asymptotic behaviour of concomitants of order statistics.

\subsection{Approximation of Excess Distribution}

In the next theorem we discuss convergence in the distribution of the scaled excesses $(K=\{1, \ldots, k\})$. The case $|K|<k$ is dealt with separately in Corollary 5.3

Theorem 5.1 Let $F_{k}, \boldsymbol{X}, \boldsymbol{t}, \boldsymbol{t}_{n}, n \geq 1$, and $I \subset\{1, \ldots, k\}$ satisfy the assumptions of Theorem 3.1 if $\omega=\infty$ or the assumptions of Theorem 3.3 in the case $\omega=1$. If $J$ is non-empty define $\widetilde{\boldsymbol{u}}_{J}$ as in Theorem 3.1, and let $\boldsymbol{v}_{n}, n \geq 1$ be a sequence of vectors as in Eq. 3.18. Then we have

$$
\boldsymbol{v}_{n} \boldsymbol{X}_{\left[t_{n}\right]} \stackrel{d}{\rightarrow} \boldsymbol{W}, \quad n \rightarrow \infty,
$$


where $\boldsymbol{W}$ is a random vector in $\mathbb{R}^{k}$ such that $\boldsymbol{W}_{I}$ has independent components with stochastic representation

$$
W_{i} \stackrel{d}{=} \mathcal{E}_{\mu_{i}}, \quad \mu_{i}:=\left(\boldsymbol{t}_{I}^{\top} \Sigma_{I I}^{-1} \boldsymbol{e}_{i}\right)^{-1} \in(0, \infty), \quad \forall i \in I,
$$

where $\mathcal{E}_{\mu_{i}}$ is an Exponential random variable with mean $\mu_{i}$.

If $|J|>0$ then $\boldsymbol{W}_{I}$ is independent of $\boldsymbol{W}_{J}$ which is defined by

$$
\boldsymbol{P}\left\{\boldsymbol{W}_{J}>\boldsymbol{x}_{J}\right\}=\frac{\boldsymbol{P}\left\{\boldsymbol{Z}_{J}>\widetilde{\boldsymbol{u}}_{J}+\boldsymbol{x}_{J} \mid \boldsymbol{Z}_{I}=\mathbf{0}_{I}\right\}}{\boldsymbol{P}\left\{\boldsymbol{Z}_{J}>\widetilde{\boldsymbol{u}}_{J} \mid \boldsymbol{Z}_{I}=\mathbf{0}_{I}\right\}}, \quad \forall \boldsymbol{x} \in \mathbb{R}^{k}: \boldsymbol{x}_{J} \in[0, \infty)^{|J|},
$$

where $\boldsymbol{Z}$ is a standard Gaussian random vector in $\mathbb{R}^{k}$ with covariance matrix $A^{\top} A$.

In the case that the index set $I$ in Theorem 5.1 has less than $k$ elements (recall $I$ cannot be empty), the appearance of $\widetilde{\boldsymbol{u}}_{J}$ in the limit above can be removed using another sequence of thresholds. This is shown in the following corollary.

Corollary 5.2 Let $\boldsymbol{X}, I, \boldsymbol{W}_{I}, \boldsymbol{Z}, \boldsymbol{t}, \boldsymbol{t}_{n}, \boldsymbol{v}_{n}, n \geq 1$, be as in Theorem 5.1. Suppose that $J=\{1, \ldots, k\} \backslash I$ is non-empty. Define for $n \geq 1$ the vector $t_{n}^{*}$ in $\mathbb{R}^{k}$ with components $\boldsymbol{t}_{n, I}^{*}:=\boldsymbol{t}_{n, I}, \boldsymbol{t}_{n, J}^{*}:=\Sigma_{J I} \Sigma_{I I}^{-1} \boldsymbol{t}_{n, I}$. Then we have

$$
\boldsymbol{v}_{n} \boldsymbol{X}_{\left[t_{n}^{*}\right]} \stackrel{d}{\rightarrow} \boldsymbol{W}^{*}, \quad n \rightarrow \infty,
$$

where $\boldsymbol{W}^{*}$ is a random vector in $\mathbb{R}^{k}$ with independent components $\boldsymbol{W}_{I}^{*}, \boldsymbol{W}_{J}^{*}$ which have stochastic representation

$$
\boldsymbol{W}_{I}^{*} \stackrel{d}{=} \boldsymbol{W}_{I}, \quad \boldsymbol{W}_{J}^{*} \stackrel{d}{=} \boldsymbol{Y}_{J} \mid \boldsymbol{Y}_{J}>\mathbf{0}_{J}, \quad \text { with } \boldsymbol{Y}_{J} \stackrel{d}{=} \boldsymbol{Z}_{J} \mid \boldsymbol{Z}_{I}=\mathbf{0}_{I} .
$$

Using our new asymptotic results it is possibly to study the asymptotic behaviour of the sequence of partial excesses $\boldsymbol{X}_{\left[\boldsymbol{t}_{n} ; K\right]}$ for any non-empty set $K \subset\{1, \ldots, k\}$. Convergence in distributions of the scaled partial excesses is obtained in the next corollary considering $\boldsymbol{t}_{n}^{*}$ and $I$ as in Corollary 5.2.

Corollary 5.3 Under the assumptions of Corollary 5.2 we have the convergence in the distribution

$$
\boldsymbol{v}_{n} \boldsymbol{X}_{\left[t_{n}^{*} ; I\right]} \stackrel{d}{\rightarrow} \widetilde{\boldsymbol{W}}, \quad n \rightarrow \infty,
$$

with $\widetilde{\boldsymbol{W}}$ a random vector in $\mathbb{R}^{k}$ such that

$$
\widetilde{\boldsymbol{W}}_{I} \stackrel{d}{=} \boldsymbol{W}_{I}, \quad \text { and } \widetilde{\boldsymbol{W}}_{J} \stackrel{d}{=} \boldsymbol{Z}_{J} \mid \boldsymbol{Z}_{I}=\mathbf{0}_{I} .
$$

Furthermore $\widetilde{\boldsymbol{W}}_{I}$ and $\widetilde{\boldsymbol{W}}_{J}$ are independent.

The above asymptotic result extends for $k \geq 2$ Theorem 12.4.1 in Berman (1992). See also Lemma 8.2 in Berman (1982), Theorem 4.1 in Berman (1983), and Theorem 3.4 in Hashorva (2006a). In the latter article for any two 
non-empty disjoint index sets $I, J$ with $I \cup J=\{1, \ldots, k\}$ a Gaussian approximation of the distribution function of $\boldsymbol{X}_{J} \mid \boldsymbol{X}_{I}=\boldsymbol{t}_{n, I}$ is obtained by letting $\boldsymbol{t}_{n, I}$ approach a boundary point.

\subsection{Conditional Limiting Distribution}

That result can be strengthen (for the case $|I \cup J|<k$ ) to convergence of density functions as shown in the next theorem.

Theorem 5.4 Let $A, k, F_{k}, R_{k}, \boldsymbol{X}, \boldsymbol{Z}$ be as in Theorem 3.1 and let $\boldsymbol{t}_{n}, n \geq 1$ be given vectors in $\mathbb{I}^{k} \backslash(-\infty, 0]^{k}$ such that $\left\|\boldsymbol{t}_{n}\right\|<\omega, n \geq 1$ with $\omega \in(0, \infty]$ the upper endpoint of $F_{k}$. For two given non-empty disjoint index sets $I, J$ with $I \cup$ $J \subset\{1, \ldots, k\}$ define a sequence of random vectors $\boldsymbol{V}_{n, J}, n \geq 1$ by the stochastic representation

$$
\boldsymbol{V}_{n, J} \stackrel{d}{=}\left(\frac{\beta_{n}}{\alpha_{n}}\right)^{1 / 2}\left(\boldsymbol{X}_{J}-\Sigma_{J I} \Sigma_{I I}^{-1} \boldsymbol{t}_{n, I}\right) \mid \boldsymbol{X}_{I}=\boldsymbol{t}_{n, I}, \quad \alpha_{n}:=\left\|\boldsymbol{t}_{n, I}\right\|, \beta_{n}:=w\left(\alpha_{n}\right), \quad n \geq 1 .
$$

Suppose that $\mathcal{M}_{R_{k}}$ has at least two elements and put $m:=|I|<k$. Then $\boldsymbol{V}_{n, J}, n \geq 1$ possesses density function $h_{n, J}$ given by

$$
\begin{aligned}
h_{n, J}\left(\boldsymbol{x}_{J}\right)= & \left(\frac{\alpha_{n}}{\beta_{n}}\right)^{(k-m) / 2} \frac{\Gamma(d / 2) \Gamma((k-m) / 2)}{\Gamma((d-k) / 2) \Gamma(k / 2) \pi^{(k-m) / 2}\left|\left(\Sigma^{-1}\right)_{J J}\right|^{1 / 2}} \\
& \times \frac{\int_{\left\|\boldsymbol{x}_{n}\right\|}^{\infty}\left(r^{2}-\left\|\boldsymbol{x}_{n}\right\|^{2}\right)^{(d-k) / 2-1} r^{-(d-2)} d F_{d}(r)}{\int_{\alpha_{n}}^{\infty}\left(r^{2}-\alpha_{n}^{2}\right)^{(k-m) / 2-1} r^{-(k-2)} d F_{k}(r)},
\end{aligned}
$$

with $F_{d}, d>k$ the distribution function of $R_{d} \in \mathcal{M}_{R_{k}}$, and

$\boldsymbol{x}_{n} \in \mathbb{R}^{k}: \quad \boldsymbol{x}_{n, I}:=\boldsymbol{t}_{n, I}, \quad \boldsymbol{x}_{n, J}:=\left(\beta_{n} / \alpha_{n}\right)^{1 / 2} \boldsymbol{x}_{J}+\Sigma_{J I} \Sigma_{I I}^{-1} \boldsymbol{t}_{n, I}, \quad n \geq 1, \quad \boldsymbol{x} \in \mathbb{R}^{k}$.

If further $F_{d} \in \operatorname{MDA}(\Lambda, w)$ and $\lim _{n \rightarrow \infty} \alpha_{n}=\omega$, then

$$
h_{n, J}\left(\boldsymbol{x}_{J}\right) \rightarrow \varphi\left(\boldsymbol{x}_{J}\right), \quad n \rightarrow \infty
$$

holds locally uniformly in $\mathbb{I R}^{m}$ with $\varphi$ the density function of the Gaussian random vector $\boldsymbol{Z}_{J} \mid \boldsymbol{Z}_{I}=\mathbf{0}_{I}$.

Recalling the fact that for a bivariate random vector the conditional density is given in terms of the joint density function and the marginal density function of the conditioning random variable we show in the next corollary that the marginal distribution functions of Type I elliptical random vectors satisfy the Von Mises condition.

Corollary 5.5 Let $\boldsymbol{X} \stackrel{d}{=} A^{\top} R \boldsymbol{U}_{k}$ be an elliptical random vector in $\mathbb{R}^{k}, k \geq 3$ with associated random radius $R \sim F$, and non-singular square matrix $A$ such 
that $\Sigma:=A^{\top} A$. If the distribution function $F$ satisfies Eq. 3.7 with the scaling function $w$ and upper endpoint $\omega \in(0, \infty]$, then we have

$$
w(t)=\frac{G^{\prime}(t)}{1-G(t)}, \quad \forall t \in(0, \omega),
$$

with $G$ the distribution function of $X_{1}$ and $G^{\prime}$ its density function.

\subsection{Concomitants of Order Statistics}

In this section we provide an asymptotic result for the distribution of concomitants of order statistics. For simplicity we discuss next only the bivariate case, where $(X, Y),\left(X_{i}, Y_{i}\right), 1 \leq i \geq 1$ are independent bivariate elliptical random vectors with common distribution function $H$. By ordering the pairs from the order statistics $X_{1: n} \leq \cdots \leq X_{n: n}, n \geq 1$ another sequence of random variables $Y_{[i: n]}, 1 \leq i \leq n$ is obtained where $Y_{[i: n]}$ is the second component of the pair with first component the $i$-th order statistics $X_{i: n}$. There are several important applications of concomitants, we mention here the one on selection procedures, where individuals are picked up upon information on their $X$-values.

Basic distributional and asymptotical results on the topic can be found in Gale (1980), Eddy and Gale (1981), Galambos (1987), Nagaraja and David (1994), Joshi and Nagaraja (1995), Ledford and Twan (1998) among several others.

Referring to Kaufmann and Reiss (1992) we have for $i=1, \ldots, n$

$$
\boldsymbol{P}\left\{Y_{[n-i+1: n]} \leq y, \ldots, Y_{[n: n]} \leq y\right\}=\int_{-\infty}^{\infty} \boldsymbol{P}\left\{Y_{1} \leq y \mid X_{1}>x\right\} d G_{n-i: n}(x), \quad y \in \mathbb{R},
$$

with $G_{n-i: n}$ the distribution function of $X_{n-i: n}$, indicating that the distribution function of the concomitants is closely related to the distribution function of the upper order statistics.

This important fact paves the way for asymptotic results related to the extreme value theory, see for instance Ledford and Twan (1998). Following the ideas in the aforementioned paper we discuss briefly the case that $X$ has distribution function $G$ in the Gumbel max-domain of attraction implying that for any integer $i \geq 0$

$$
\left(X_{n-i: n}-b_{n}\right) / a_{n} \stackrel{d}{\rightarrow} \eta_{i}, \quad n \rightarrow \infty
$$

is valid with $a_{n}>0, b_{n}, n \geq 1$ constants (see e.g., Falk et al. 2004) and limiting random variable $\eta_{i}$ which possesses the density function $\Lambda^{\prime}(x)(-\ln \Lambda(x))^{i} / i$ ! . ( $\Lambda^{\prime}$ denotes the density function of the unit Gumbel distribution $\Lambda$ ).

We state next the asymptotic result for Type I bivariate elliptical random vectors. Multivariate extension in the spirit of Gale (1980) and Eddy and Gale (1981) can be shown using our results in Section 5.

Theorem 5.6 Let $\left(S_{1}, S_{2}\right)$ be a bivariate spherical random vector with associate random radius $R>0$, and let the random variable $U \sim \Lambda$ be independent 
of the standard Gaussian random variable $Z$. Define $\left(X_{n}, Y_{n}\right), n \geq 1$ via the stochastic representation $\left(X_{n}, Y_{n}\right) \stackrel{d}{=}\left(S_{1}, \rho S_{1}+\sqrt{1-\rho^{2}} S_{2}\right), \rho \in(-1,1)$. If the distribution function $F$ of the associated random radius $R$ is in the Gumbel maxdomain of attraction with the scaling function $w$, then we have the convergence in distribution

$$
\left(w\left(b_{n}\right)\left(X_{n: n}-b_{n}\right), \frac{Y_{[n: n]}-\rho b_{n}}{\sqrt{\left(1-\rho^{2}\right) b_{n} / w\left(b_{n}\right)}}\right) \stackrel{d}{\rightarrow}(U, Z), \quad n \rightarrow \infty,
$$

where $b_{n}:=G^{-1}(1-1 / n)$ with $G^{-1}$ the generalised inverse of the distribution function of $S_{1}$.

The asymptotic result above agrees with the one stated in Theorem 5.1 of Gale (1980), where the associated random radius has (asymptotically) exponential tails. See also Eddy and Gale (1981); Ledford and Twan (1998).

\section{Examples}

We consider next three illustrating examples. In the first example we retrieve the result for the Gaussian case, followed by two examples dealing with the specifics of the bivariate setup.

Example 6.1 (Gaussian Tails) Let $\boldsymbol{X}$ be a standard Gaussian random vector with positive definite covariance matrix $\Sigma=A^{\top} A, A \in \mathbb{R}^{k \times k}$. $\boldsymbol{X}$ has stochastic representation (Eq. 1.2) with $\mathbb{R}_{k}>0$ such that $R_{k}^{2}$ is chi-squared distributed with $k$ degrees of freedom. We have

$$
\boldsymbol{P}\left\{R_{k}>u\right\}=\frac{(1+o(1)) u^{k-2}}{2^{k / 2-1} \Gamma(k / 2)} \exp \left(-u^{2} / 2\right), \quad u \rightarrow \infty
$$

and Eq. 3.7 holds with $w(u):=u, u>0$, hence $F_{k} \in M D A(\Lambda, w)$ holds. Let $\boldsymbol{t}_{n}, n \geq 1$ be a sequence of thresholds in $\mathbb{R}^{k} \backslash(-\infty, 0]^{k}$ such that Eq. 3.10 is satisfied, and if $|I|<k$ assume additionally $\lim _{n \rightarrow \infty}\left(\boldsymbol{t}_{n, J}-\Sigma_{J I} \Sigma_{I I}^{-1} \boldsymbol{t}_{n, I}\right)=\widetilde{\boldsymbol{u}}_{J}$. In view of our results above we obtain the asymptotic expansion

$$
\begin{aligned}
\boldsymbol{P}\left\{\boldsymbol{X}>\boldsymbol{t}_{n}\right\}= & (1+o(1)) \frac{\boldsymbol{P}\left\{\boldsymbol{Z}_{J}>\widetilde{\boldsymbol{u}}_{J} \mid \boldsymbol{Z}_{I}=\mathbf{0}_{I}\right\}}{(2 \pi)^{|I| / 2}\left|\Sigma_{I I}\right|^{1 / 2} \prod_{i \in I} \boldsymbol{t}_{n, I}^{\top} \Sigma_{I I}^{-1} \boldsymbol{e}_{i}} \\
& \times \exp \left(-\alpha_{n}^{2} / 2\right), \quad n \rightarrow \infty .
\end{aligned}
$$

Eq. 6.37 is shown in Corollary 4.1 of Hashorva and Hüsler (2003).

Example 6.2 (Bivariate Tails) Consider $\boldsymbol{X}=R_{2} A^{\top} \boldsymbol{U}_{2}$ a bivariate elliptical random vector of Type I. Let $\omega$ be the upper endpoint of the distribution function $F_{2}$ of $R_{2}$. We consider both cases $\omega=1$ or $\omega=\infty$. Assume that $F_{2} \in$ $\operatorname{MDA}(\Lambda, w)$ and $\Sigma:=A^{\top} A \in \mathbb{R}^{2 \times 2}$ is such that $\sigma_{11}=\sigma_{22}=1$ and $\sigma_{12}=\sigma_{21}=$ : 
$\rho \in(-1,1)$. Let $t_{n}, n \in \mathbb{N}$ be positive constants converging to $\omega$ as $n \rightarrow \infty$. The solution of the quadratic problem $\mathcal{P}\left(\Sigma^{-1}, t_{n} \boldsymbol{a}\right)$ with $\boldsymbol{a}=(1, a)^{\top}, a \in(-1,1]$ depends only on $a$. We have three possible cases:

i) Case $\rho<a$ : $\quad t_{n} \boldsymbol{a}$ is the solution of $\mathcal{P}\left(\Sigma^{-1}, t_{n} \boldsymbol{a}\right)$ for any $n \geq 1$. The minimal index set $I$ is $\{1,2\}$ and $J$ is empty. Furthermore, for any $n \geq 1$

$$
\alpha_{n}=t_{n}\left(\left(1-2 \rho a+a^{2}\right) /\left(1-\rho^{2}\right)\right)^{1 / 2}=t_{n} b_{*}, \quad b_{*}:=\left\|\boldsymbol{a}_{I}\right\|>0
$$

and $\boldsymbol{t}_{I}:=\boldsymbol{a}_{I} / b_{*}$. Hence $t_{n} \boldsymbol{a}, n \geq 1$ satisfies the conditions of Theorem 3.1 if $\omega=\infty$, consequently

$$
\boldsymbol{P}\left\{\boldsymbol{X}>t_{n} \boldsymbol{a}\right\}=(1+o(1)) \frac{\left(1-\rho^{2}\right)^{3 / 2}}{2 \pi(1-\rho a)(a-\rho)}\left(t_{n} w\left(b_{*} t_{n}\right)\right)^{-1}\left(1-F_{2}\left(b_{*} t_{n}\right)\right), n \rightarrow \infty .
$$

If $\omega=1$ the above asymptotic expansion holds provided $\|\boldsymbol{a}\|=1$.

ii) Case $\rho=a \in(-1,1)$ : For any $n \in \mathbb{N}$ we have $I=\{1\}, J=\{2\}$. Further $b_{*}:=\left\|\boldsymbol{a}_{I}\right\|=1, \boldsymbol{t}_{I}=1, \alpha_{n}=t_{n}, n \geq 1$. Condition 3.11 holds with $\widetilde{\boldsymbol{u}}_{J}=0$, hence we obtain

$$
\boldsymbol{P}\left\{\boldsymbol{X}>t_{n} \boldsymbol{a}\right\}=(1+o(1))(8 \pi)^{-1 / 2}\left(t_{n} w\left(t_{n}\right)\right)^{-1 / 2}\left(1-F_{2}\left(t_{n}\right)\right), \quad n \rightarrow \infty .
$$

iii) Case $\rho \in(a, 1), a<1$ : We have $I=\{1\}, J=\{2\},\left\|\boldsymbol{a}_{I}\right\|=1, \boldsymbol{t}_{I}=1$ and $\alpha_{n}=t_{n}, n \geq 1$. (Eq. 3.11) holds with $\widetilde{\boldsymbol{u}}_{J}=-\infty$, hence we have

$$
\boldsymbol{P}\left\{\boldsymbol{X}>t_{n} \boldsymbol{a}\right\}=(1+o(1))(2 \pi)^{-1 / 2}\left(t_{n} w\left(t_{n}\right)\right)^{-1 / 2}\left(1-F_{2}\left(t_{n}\right)\right), \quad n \rightarrow \infty .
$$

As in the above example we consider next approximation of the density function, approximation of the excess distribution, and some conditional limiting results.

Example 6.3 Let $\boldsymbol{a}, \boldsymbol{X}, \Sigma, t_{n}, n \geq 1$ be as in the previous example, and assume for simplicity that $\|\boldsymbol{a}\|=1$.

(i) Case $\rho \in(-1, a)$ : In view of Theorem 5.1

$$
\left(w\left(t_{n}\right)\left(X_{1}-t_{n} a_{1}\right), w\left(t_{n}\right)\left(X_{1}-t_{n} a_{2}\right)\right) \mid \boldsymbol{X}>t_{n} \boldsymbol{a} \stackrel{d}{\rightarrow}\left(W_{1}, W_{2}\right), \quad n \rightarrow \infty,
$$

where $W_{i} \stackrel{d}{=} \mathcal{E}_{\mu_{i}}, i=1,2$ with $\mu_{i}=1 /\left(\boldsymbol{a} \Sigma^{-1} \boldsymbol{e}_{i}\right) \in(0, \infty)$.

(ii) Case $\rho \in[a, 1)$ : We have $I_{n}=I=\{1\}, n \geq 1$. (Recall we assume $\left.\lim _{n \rightarrow \infty} t_{n}=\omega \in\{1, \infty\}\right)$. Let $\Phi$ denote a standard Gaussian distribution on IR. Applying Corollary 5.2 we have

$$
\left(w\left(t_{n}\right)\left(X_{1}-t_{1}\right),\left(\frac{w\left(t_{n}\right)}{t_{n}}\right)^{1 / 2}\left(X_{2}-\rho t_{n}\right)\right) \mid X_{1}>t_{n} a_{1}, X_{2}>t_{n} a_{2} \stackrel{d}{\rightarrow}\left(W_{1}, W_{2}\right), \quad n \rightarrow \infty,
$$


with $W_{1} \stackrel{d}{=} \mathcal{E}_{1}$, and $W_{2}>0$ has distribution function $2\left[\Phi\left(x\left(1-\rho^{2}\right)^{-1 / 2}\right)-\right.$ $1 / 2$ ], $x>0$ being further independent of $W_{1}$. A different result is obtained applying Corollary 5.3. We have

$$
\left(w\left(t_{n}\right)\left(X_{1}-t_{n}\right),\left(\frac{w\left(t_{n}\right)}{t_{n}}\right)^{1 / 2}\left(X_{2}-\rho t_{n}\right)\right) \mid X_{1}>t_{n} \stackrel{d}{\rightarrow}\left(W_{1}, W_{2}^{*}\right), \quad n \rightarrow \infty,
$$

with $W_{1}$ as above and $W_{2}^{*} \sim \Phi$ independent of $W_{1}$. See Eq. 3.12 in Hashorva (2006a) for a similar result. Clearly, Eq. 6.38 implies

$$
\left(\frac{w\left(t_{n}\right)}{t_{n}}\right)^{1 / 2}\left(X_{2}-\rho t_{n}\right) \mid X_{1}>t_{n} \stackrel{d}{\rightarrow} W_{2}^{*}, \quad n \rightarrow \infty,
$$

which is shown in Theorem 4.1 in Berman (1983) and Theorem 12.4.1 in Berman (1992). See also Gale (1980); Eddy and Gale (1981). To this end we remark that (see Hashorva 2006a)

$$
\left(\frac{w\left(t_{n}\right)}{t_{n}}\right)^{1 / 2}\left(X_{2}-\rho t_{n}\right) \mid X_{1}=t_{n} \stackrel{d}{\rightarrow} W_{2}^{*}, \quad n \rightarrow \infty,
$$

which actually implies Eq. 6.39. Moreover, under the assumptions of Theorem 5.4 we have that the convergence can be stated for the corresponding density functions.

\section{Related Results and Proofs}

Initially we present two results related to quadratic programming.

Proposition 7.1 [Proposition 2.1 of Hashorva (2005a)] Let $\Sigma \in \mathbb{R}^{k \times k}, k \geq 2$, be a positive definite correlation matrix and let $\boldsymbol{a} \in \mathbb{R}^{k} \backslash(-\infty, 0]^{k}$ be a fixed vector. Then the quadratic programming problem

$$
\mathcal{P}\left(\Sigma^{-1}, \boldsymbol{a}\right) \text { : minimise }\|\boldsymbol{x}\|^{2} \text { under the linear constraint } \boldsymbol{x} \geq \boldsymbol{a}
$$

has a unique solution $\widetilde{\boldsymbol{a}}$ and there exists a unique non-empty index set $I \subset$ $\{1, \ldots, k\}$ so that

$$
\begin{aligned}
\tilde{\boldsymbol{a}}_{I}=\boldsymbol{a}_{I}>\mathbf{0}_{I}, \quad \text { and if } J:=\{1, \ldots, d\} \backslash I \neq \emptyset, \text { then } \tilde{\boldsymbol{a}}_{J} & =\Sigma_{J I} \Sigma_{I I}^{-1} \boldsymbol{a}_{I} \geq \boldsymbol{a}_{J}, \\
\Sigma_{I I}^{-1} \boldsymbol{a}_{I} & >\mathbf{0}_{I}, \\
\min _{\boldsymbol{x} \geq \boldsymbol{a}}\|\boldsymbol{x}\|^{2}=\min _{\boldsymbol{x} \geq \boldsymbol{a}} \boldsymbol{x}^{\top} \Sigma^{-1} \boldsymbol{x}=\|\tilde{\boldsymbol{a}}\|^{2}=\left\|\boldsymbol{a}_{I}\right\|^{2} & =\boldsymbol{a}_{I}^{\top} \Sigma_{I I}^{-1} \boldsymbol{a}_{I}>0 .
\end{aligned}
$$

Furthermore, for any $\boldsymbol{x} \in \mathbb{R}^{k}$ we have

$$
\boldsymbol{x}^{\top} \Sigma^{-1} \widetilde{\boldsymbol{a}}=\boldsymbol{x}_{I}^{\top} \Sigma_{I I}^{-1} \widetilde{\boldsymbol{a}}_{I}=\boldsymbol{x}_{I}^{\top} \Sigma_{I I}^{-1} \boldsymbol{a}_{I}=\sum_{i \in I} x_{i} h_{i},
$$

with $h_{i}:=\boldsymbol{e}_{i}^{\top} \Sigma_{I I}^{-1} \boldsymbol{a}_{I}>0, \forall i \in$ I. If $\boldsymbol{a}=c \mathbf{1}, c \in(0, \infty)$, we have $2 \leq|I| \leq k$. 
In view of the above proposition there are situations where the solution of the quadratic programming problem $\mathcal{P}\left(\Sigma^{-1}, \boldsymbol{a}\right)$ is the vector $\boldsymbol{a}$ if it satisfies the Savage condition (Eq. 2.6). Next, we show that for a given positive definite matrix $\Sigma$ it is possible to find $\boldsymbol{a} \in \mathbb{I}^{k}$ such that Eq. 2.6 is not valid, and in particular the solution of $\mathcal{P}\left(\Sigma^{-1}, \boldsymbol{a}\right)$ is not the vector $\boldsymbol{a}$.

Proposition 7.2 Let $\Sigma$ be as in Proposition 7.1 and let $K$ be a non-empty subset of $\{1, \ldots, k\}$ with $|K|=m<k$. For a given vector $\boldsymbol{b} \in \mathbb{R}^{k}$ let $\boldsymbol{a} \in \mathbb{R}^{k}$ be another vector such that

$$
\boldsymbol{a}_{K}=\boldsymbol{b}_{K}, \quad \boldsymbol{a}_{L} \in \mathbb{R}^{k-m}: \boldsymbol{a}_{L} \leq \Sigma_{L K} \Sigma_{K K}^{-1} \boldsymbol{b}_{K}, \quad \text { with } L:=\{1, \ldots, k\} \backslash K .
$$

The quadratic programming problem $\mathcal{P}\left(\Sigma^{-1}\right.$, a $)$ defined in Eq. 7.1 has a unique solution $\tilde{\boldsymbol{a}}$ determined by a unique non-empty index set $I \subset K$ such that Eqs. 7.40, 7.41 and 7.42 hold. In the special case $\boldsymbol{a}_{L}=\Sigma_{L K} \Sigma_{K K}^{-1} \boldsymbol{b}_{K}$

$$
\|\boldsymbol{a}+\boldsymbol{x}\|^{2}=\left\|\boldsymbol{a}_{K}\right\|^{2}+2 \boldsymbol{a}_{K}^{\top} \Sigma_{K K}^{-1} \boldsymbol{x}_{K}+\|\boldsymbol{x}\|^{2}
$$

is valid for any $\boldsymbol{x} \in \mathbb{R}^{k}$.

Proof Utilising a probabilistic proof we show first that $I \subset K$. Let $\boldsymbol{Y}$ be a standard Gaussian random vector in $\mathbb{R}^{k}$ with correlation matrix $\Sigma$. In the light of Theorem 3.4 in Hashorva (2005a) there exists a unique non-empty index set $M \subset K$ which defines the unique solution of $\mathcal{P}\left(\Sigma_{K K}^{-1}, \boldsymbol{a}_{K}\right)$ such that

$$
\lim _{t \rightarrow \infty} \frac{\boldsymbol{P}\left\{\boldsymbol{X}_{K}>t \boldsymbol{a}_{K}\right\}}{\boldsymbol{P}\left\{\boldsymbol{X}_{M}>t \boldsymbol{a}_{M}\right\}}=c_{1} \in(0, \infty)
$$

holds. By the definition of $\boldsymbol{a}_{L}$ it follows using Theorem 3.1 in Hashorva (2005a) that

$$
\lim _{t \rightarrow \infty} \frac{\boldsymbol{P}\{\boldsymbol{X}>t \boldsymbol{a}\}}{\boldsymbol{P}\left\{\boldsymbol{X}_{K}>t \boldsymbol{a}_{K}\right\}}=c_{2} \in(0, \infty),
$$

consequently

$$
\lim _{t \rightarrow \infty} \frac{\boldsymbol{P}\{\boldsymbol{X}>t \boldsymbol{a}\}}{\boldsymbol{P}\left\{\boldsymbol{X}_{M}>t \boldsymbol{a}_{M}\right\}}=c_{1} c_{2}>0,
$$

which implies [using again Theorem 3.4 in Hashorva (2005a)] that the minimal index set $I$ related to $\mathcal{P}\left(\Sigma^{-1}, \boldsymbol{a}\right)$ must be a subset of $M$, hence $I \subset K$.

Note in passing that if $\Sigma_{K K}^{-1} \boldsymbol{a}_{K}>\mathbf{0}_{K}$, then the vector $\tilde{\boldsymbol{a}}$ defined by $\tilde{\boldsymbol{a}}_{K}=\boldsymbol{a}_{K}$ and $\tilde{\boldsymbol{a}}_{L}=\Sigma_{L K} \Sigma_{K K}^{-1} \boldsymbol{b}_{K}$ is the unique solution of $\mathcal{P}\left(\Sigma^{-1}, \boldsymbol{a}\right)$, implying that $I=K$.

Next, we prove Eq. 7.44. Denote the inverse matrix of $\Sigma$ (which exists) by $B$. Since $\Sigma$ is positive definite we have

$$
B_{L L}^{-1} B_{L K}=-\Sigma_{L K} \Sigma_{K K}^{-1} .
$$

Consequently we obtain for any $\boldsymbol{y} \in \mathbb{R}^{k}$

$$
\begin{aligned}
\|\boldsymbol{y}\|^{2} & =\boldsymbol{y}^{\top} \Sigma^{-1} \boldsymbol{y}=\boldsymbol{y}_{K}^{\top} \Sigma_{I I}^{-1} \boldsymbol{y}_{K}+\left(\boldsymbol{y}_{L}+B_{L L}^{-1} B_{L K} \boldsymbol{y}_{I}\right)^{\top} B_{L L}^{-1}\left(\boldsymbol{y}_{L}+B_{L L}^{-1} B_{L K} \boldsymbol{y}_{K}\right) \\
& =\left\|\boldsymbol{y}_{K}\right\|^{2}+\left(\boldsymbol{y}_{L}-\Sigma_{L K} \Sigma_{K K}^{-1} \boldsymbol{y}_{K}\right)^{\top} B_{L L}^{-1}\left(\boldsymbol{y}_{L}-\Sigma_{L K} \Sigma_{K K}^{-1} \boldsymbol{y}_{K}\right) .
\end{aligned}
$$


In our case we have for any $\boldsymbol{x} \in \mathbb{R}^{k}$

$$
\begin{aligned}
\|\boldsymbol{a}+\boldsymbol{x}\|^{2}= & \left\|\boldsymbol{a}_{K}+\boldsymbol{x}_{K}\right\|^{2} \\
& +\left((\boldsymbol{a}+\boldsymbol{x})_{L}-\Sigma_{L K} \Sigma_{K K}^{-1}(\boldsymbol{a}+\boldsymbol{x})_{K}\right)^{\top} B_{L L}^{-1}\left((\boldsymbol{a}+\boldsymbol{x})_{L}-\Sigma_{L K} \Sigma_{K K}^{-1}(\boldsymbol{a}+\boldsymbol{x})_{K}\right) \\
= & \left\|\boldsymbol{a}_{K}\right\|^{2}+2 \boldsymbol{a}_{K}^{\top} \Sigma_{K K}^{-1} \boldsymbol{x}_{K}+\left\|\boldsymbol{x}_{K}\right\|^{2} \\
& +\left(\boldsymbol{x}_{L}-\Sigma_{L K} \Sigma_{K K}^{-1} \boldsymbol{x}_{K}\right)^{\top} B_{L L}^{-1}\left(\boldsymbol{x}_{L}-\Sigma_{L K} \Sigma_{K K}^{-1} \boldsymbol{x}_{K}\right) \\
= & \left\|\boldsymbol{a}_{K}\right\|^{2}+2 \boldsymbol{a}_{K}^{\top} \Sigma_{K K}^{-1} \boldsymbol{x}_{k}+\|\boldsymbol{x}\|^{2} .
\end{aligned}
$$

This completes the proof.

Lemma 7.3 Let $A \in \mathbb{R}^{k \times k}, k \geq 2$, be a non-singular matrix and let $\boldsymbol{X} \stackrel{d}{=}$ $R_{k} A^{\top} \boldsymbol{U}_{k}, \boldsymbol{Y} \stackrel{d}{=} R_{k}^{*} A^{\top} \boldsymbol{U}_{k}$ be two random vectors in $\mathbb{I}^{k}$ such that $R_{k}, R_{k}^{*}$ are almost surely positive being further independent of $\boldsymbol{U}_{k}$. Let $\boldsymbol{t}_{n}, n \geq 1$ be a sequence of thresholds in $\mathbb{R}^{k} \backslash(-\infty, 0]^{k}$ satisfying Eq. 3.10. Suppose that

$$
\lim _{u \uparrow \omega} \frac{\boldsymbol{P}\left\{R_{k}>u\right\}}{\boldsymbol{P}\left\{R_{k}^{*}>u\right\}}=c \in(0, \infty)
$$

holds with $\omega=\infty$ the upper endpoint of the distribution function of $R_{k}$. Then we have

$$
\lim _{n \rightarrow \infty} \frac{\boldsymbol{P}\left\{\boldsymbol{X}>\boldsymbol{t}_{n}\right\}}{\boldsymbol{P}\left\{\boldsymbol{Y}>\boldsymbol{t}_{n}\right\}}=c .
$$

Proof Denote by $G$ the distribution function of $A^{\top} \boldsymbol{U}_{k}$. We assume for simplicity that $\boldsymbol{t}_{n}$ is positive for all $n$ large. The other case that $\boldsymbol{t}_{n}$ has at least one negative component follows with similar arguments using Eq. 3.10, the fact that $\boldsymbol{U}_{k}$ has continuous distribution function and the rapid variation of $1-F_{k}$, i.e. for all $a>1$

$$
\lim _{x \rightarrow \infty} \frac{1-F_{k}(a x)}{1-F_{k}(x)}=0 .
$$

See Resnick (1987) for the basic properties of rapidly varying distribution functions. Next, since $R_{k}>0$ almost surely being further independent of $A^{\top} \boldsymbol{U}_{k}$ we obtain for all $n$ large

$$
\begin{aligned}
\boldsymbol{P}\left\{\boldsymbol{X}>\boldsymbol{t}_{n}\right\} & =\int_{\boldsymbol{y}>\mathbf{0}} \boldsymbol{P}\left\{R_{k}>\boldsymbol{t}_{n} / \boldsymbol{y}\right\} d G(\boldsymbol{y}) \\
& =\int_{\boldsymbol{y}>\mathbf{0}} \boldsymbol{P}\left\{R_{k}>\max _{1 \leq i \leq k}\left(t_{n i} / y_{i}\right)\right\} d G(\boldsymbol{y}) .
\end{aligned}
$$

Condition 3.10 implies $\lim _{n \rightarrow \infty} \max _{1 \leq i \leq k} t_{n i} / y_{i}=\infty$ for any $\boldsymbol{y}=\left(y_{1}, \ldots, y_{k}\right)^{\top} \in$ $(0, \infty)^{k}$, consequently

$$
\lim _{n \rightarrow \infty} \frac{\boldsymbol{P}\left\{R_{k}>\max _{1 \leq i \leq k}\left(t_{n i} / y_{i}\right)\right\}}{\boldsymbol{P}\left\{R_{k}^{*}>\max _{1 \leq i \leq k}\left(t_{n i} / y_{i}\right)\right\}}=c
$$


holds for any $y_{i}>0,1 \leq i \leq k$. Since $A$ is a non-singular matrix the random vector $A^{\top} \boldsymbol{U}_{k}$ is bounded away from the origin of $\mathbb{R}^{k}$, implying that there exits a compact set $K \subset \mathbb{R}^{k}$ such that $\boldsymbol{P}\left\{A^{\top} \boldsymbol{U}_{k} \in K\right\}=1$. The above convergence holds uniformly in $K \cap(0, \infty)^{k}$, consequently we obtain

$$
\begin{aligned}
\int_{\boldsymbol{y}>\mathbf{0}} \boldsymbol{P}\left\{R_{k}>\max _{1 \leq i \leq k}\left(t_{n i} / y_{i}\right)\right\} d G(\boldsymbol{y}) & =(1+o(1)) c \int_{\boldsymbol{y}>\mathbf{0}} \boldsymbol{P}\left\{R_{k}^{*}>\max _{1 \leq i \leq k}\left(t_{n i} / y_{i}\right)\right\} d G(\boldsymbol{y}) \\
& =(1+o(1)) \boldsymbol{P}\left\{\boldsymbol{Y}>\boldsymbol{t}_{n}\right\}, \quad n \rightarrow \infty,
\end{aligned}
$$

thus the proof is complete.

Lemma 7.4 Let $H_{0}$ be a distribution function with the upper endpoint $\omega:=$ $\sup \left\{x: H_{0}(x)<1\right\} \in(0, \infty]$. If $H_{0} \in M D A(\Lambda, w)$, then for any $\gamma \in \mathbb{R}$ there exists an univariate distribution function $H_{\gamma}$ with the upper endpoint $\omega$ defined asymptotically by

$$
1-H_{\gamma}(u)=(1+o(1))(u w(u))^{\gamma}\left[1-H_{0}(u)\right], \quad u \uparrow \omega .
$$

Furthermore, $H_{\gamma}$ is in the Gumbel max-domain of attraction with the scaling function $w$.

Proof Since $H_{0} \in M D A(\Lambda, w)$ then Eq. 3.14 implies

$$
w(u)=\frac{(1+o(1))[1-F(u)]}{\int_{u}^{\omega}[1-F(s)] d s}, \quad u \uparrow \omega .
$$

Further (see Resnick 1987) $H_{0}$ is asymptotically equivalent with a Von Mises distribution function. So it suffices to show the proof assuming that $H_{0}$ is a Von Mises distribution function with the following representation (see De Haan 1970; Resnick 1987)

$$
1-H_{0}(x)=c \exp \left(-\int_{x}^{\omega} \frac{1}{g(s)} d s\right), \quad \forall x \in(z, \omega),
$$

with $c$ a positive constant, $z<\omega$ and $g(t)$ a positive measurable function such that $\lim _{t \rightarrow \omega} g^{\prime}(t)=0$.

Let $H_{\gamma}, \gamma>0$ be a positive function defined asymptotically by Eq. 7.47. In order to complete the proof we show first that $H_{\gamma}$ is monotone non-increasing for $u$ sufficiently close to $\omega$ and vanishes to 0 as $u \uparrow \omega$.

We deal first with the case $\gamma=1$. For $u$ close enough to $\omega$ (if $\omega=\infty$ then this holds for all large $u$ ) we compute the derivative of $1-H_{1}$ using the Von Mises representation above and Eq. 3.14. We have for all $u$ close to $\omega$

$$
\left(1-H_{1}(u)\right)^{\prime}=(w(u))^{2}\left[\left(1-\frac{2 u h(u)}{1-H_{0}(u)}\right) \int_{u}^{\omega}\left(1-H_{0}(s)\right) d s-u\left(1-H_{0}(u)\right)\right],
$$

with $h:=H_{0}^{\prime}$. Since for $\omega=\infty$ [see e.g. De Haan (1970) or Resnick (1987)]

$$
\lim _{u \rightarrow \infty} \frac{u h(u)}{1-H_{0}(u)}=\infty
$$


and for $\omega<\infty$

$$
\lim _{u \uparrow \omega} \frac{(\omega-u) h(u)}{1-H_{0}(u)}=\infty
$$

we deduce that $\left(1-H_{1}(u)\right)^{\prime}$ is negative for $u$ close to $\omega$. Consequently, the function $H_{1}$ is monotone increasing for $u$ close to $\omega$. In view of Eq. 3.15, we have

$$
\lim _{u \uparrow \omega} \frac{1-H_{1}(u+x / w(u))}{1-H_{1}(u)}=\lim _{u \uparrow \omega} \frac{1-H_{0}(u+x / w(u))}{1-H_{0}(u)}=\exp (-x), \quad \forall x \in \mathbb{R},
$$

which implies

$$
\lim _{u \uparrow \omega} u\left(1-H_{1}(u)\right)=0 .
$$

Thus $H_{1}$ can be defined as an univariate distribution with the required asymptotic tail behaviour being further in the Gumbel max-domain of attraction with the scaling function $w$. Iterating it follows that $H_{\gamma}$ can be defined as an univariate distribution function for any integer $\gamma \in \mathbb{N}$. Using now the result of Theorem 12.3.1 of Berman (1992) (given in Theorem 8.2 below) it follows that $H_{\gamma}$ can be defined for any $\gamma>0$. In view of Berman's theorem it is clear that the claim holds for any $\gamma \leq 0$, thus the proof is complete.

Lemma 7.5 Let $H$ be an univariate distribution function in the Gumbel maxdomain of attraction with upper endpoint $\omega \in(0, \infty]$. Then we have for any $\alpha>-1, y \in \mathbb{R}$

$$
\lim _{u \uparrow \omega} \frac{1}{1-H(u)} \int_{y}^{\infty} s^{\alpha} d H_{u}(s)=\int_{y}^{\infty} s^{\alpha} \exp (-s) d s,
$$

with $H_{u}(s)=H(u+s / w(u)), s \in \mathbb{R}, u<\omega$.

Proof The proofs follows easily using Lemma 4.3 of Hashorva (2006a).

Lemma 7.6 Let $\alpha, H, \omega$ be as in Lemma 7.5 and let $y_{u}, z_{u} \in \mathbb{R}, u<\omega$ be constants such that $\lim _{u \uparrow \omega} y_{u}=y \in \mathbb{R}$ and $\lim _{u \uparrow \omega} z_{u}=z \in \mathbb{R}$ hold. Then we have for any $\beta \in \mathbb{R}$

$$
\begin{aligned}
& \frac{1}{1-H(u)} \int_{u+\left(z_{u}+y_{u}\right) / w(u)}^{\omega}\left[x^{2}-\left(u+z_{u} / w(u)\right)^{2}\right]^{\alpha} x^{\beta} d H(x) \\
& =(1+o(1))\left(\frac{2 u}{w(u)}\right)^{\alpha} u^{\beta} \exp (-z) \int_{y}^{\infty} x^{\alpha} \exp (-x) d x, \quad u \uparrow \omega .
\end{aligned}
$$

Proof The proof is similar to the proof of Lemma 4.5 of Hashorva (2006a) where the case $y \geq 0$ and $\beta \leq 0$ is considered.

The result of the next lemma should be known, but we could not find any similar formulation in the literature. 
Lemma 7.7 Let $H, H_{n}, n \geq 1$ be a sequence of distribution functions in $[0, \infty)$, and $f_{n}, g_{n}, n \geq 1$ be two sequences of positive measurable functions. Suppose that $H_{n}(x) \rightarrow H(x), n \rightarrow \infty$ holds for all continuity points $x$ of $H$. Assume further that locally uniformly on $[0, \infty)$ we have

$$
f_{n}(x) \rightarrow f(x), \quad \text { and } \quad g_{n}(x) \rightarrow g(x), \quad n \rightarrow \infty .
$$

If $f_{n}(x) \leq g_{n}(x), \forall x \in[K, \infty), n \geq 1$ with $K$ a positive constant and

$$
\lim _{n \rightarrow \infty} \int_{0}^{\infty} g_{n}(x) d H_{n}(x)=\int_{0}^{\infty} g(x) d H(x)<\infty,
$$

then we have

$$
\lim _{n \rightarrow \infty} \int_{0}^{\infty} f_{n}(x) d H_{n}(x)=\int_{0}^{\infty} f(x) d H(x)<\infty .
$$

Proof Since $H_{n} \rightarrow H$ weakly we have

$$
\liminf _{n \rightarrow \infty} \int_{0}^{\infty} f_{n}(x) d H_{n}(x) \geq \int_{0}^{\infty} f(x) d H(x) .
$$

By the assumptions both sequences $f_{n}, n \geq 1$ and $g_{n}, n \geq 1$ are uniformly bounded on any compact interval $[0, M], M>0$. Applying Lemma 4.2 in Hashorva (2007b) we have for any $M>0$ which is a continuity point of $H$

$$
\lim _{n \rightarrow \infty} \int_{0}^{M} f_{n}(x) d H_{n}(x)=\int_{0}^{M} f(x) d H(x)<\infty
$$

and

$$
\lim _{n \rightarrow \infty} \int_{0}^{M} g_{n}(x) d H_{n}(x)=\int_{0}^{M} g(x) d H(x)<\infty .
$$

Consequently we have

$$
\lim _{n \rightarrow \infty} \int_{M}^{\infty} g_{n}(x) d H_{n}(x)=\int_{M}^{\infty} g(x) d H(x)<\infty .
$$

In order to establish the proof it suffices to show that

$$
\limsup _{k \rightarrow \infty} \lim _{n \rightarrow \infty} \int_{M_{k}}^{\infty} f_{n}(x) d H_{n}(x)=0,
$$

with $M_{k}, k \geq 1$ a positive increasing sequence converging to the upper endpoint of the distribution function $H$ such that $M_{k}, k \geq 1$ are continuity points of $H$. We may write for all large $k$

$$
\limsup _{n \rightarrow \infty} \int_{M_{k}}^{\infty} f_{n}(x) d H_{n}(x) \leq \lim _{n \rightarrow \infty} \int_{M_{k}}^{\infty} g_{n}(x) d H_{n}(x)=\int_{M_{k}}^{\infty} g(x) d H(x),
$$

hence

$$
\underset{k \rightarrow \infty}{\limsup } \lim _{n \rightarrow \infty} \int_{M_{k}}^{\infty} f_{n}(x) d H_{n}(x) \leq \limsup _{k \rightarrow \infty} \int_{M_{k}}^{\infty} g(x) d H(x)=0,
$$

thus the proof is complete. 
Proof of Theorem 3.1 We assume for simplicity that the index set $J$ is not empty, i.e. $|I|<k$. Recall that by Proposition 7.1 we have $|I| \geq 1$. For $n>1$ let in the following $\alpha_{n}, \beta_{n}$ and $\boldsymbol{v}_{n}$ be defined as in Eqs. 3.8 and 3.18, respectively. Set $d:=k+2$ and let $F_{d}$ be the distribution function of an almost surely positive random variable $R_{d}$ defined asymptotically by

$$
1-F_{d}(u)=(1+o(1)) u w(u)\left[1-F_{k}(u)\right] \frac{\Gamma(k / 2)}{2 \Gamma(d / 2)}, \quad u \rightarrow \infty .
$$

Such a distribution function exists in view of Lemma 7.4 and $F_{d}$ has upper endpoint $\infty$. Equation 3.15 implies that $F_{d}$ satisfies Eq. 3.7 with the same scaling function $w$.

Define a random vector $\boldsymbol{W}$ in $\mathbb{R}^{d}$ via the stochastic representation $\boldsymbol{W} \stackrel{d}{=}$ $R_{d} B^{\top} \boldsymbol{U}_{d}$ where $R_{d}$ is independent of $\boldsymbol{U}_{d}, B$ is a $d \times d$ square matrix such that $\Sigma=\left(B^{\top} B\right)_{K, K}$ with $K=\{1, \ldots, k\}$. Clearly $B$ exists, and $R_{d}$ is independent of $\boldsymbol{U}_{d}$. Denote by $\boldsymbol{Y}$ the vector consisting of the first $k$ elements of $\boldsymbol{W}$. By the construction we have $\boldsymbol{Y} \stackrel{d}{=} R_{k}^{*} A^{\top} \boldsymbol{U}_{k}$, with $R_{k}^{*}>0$ almost surely being further independent of $\boldsymbol{U}_{k}$. Equation 4.21 implies $\left(R_{k}^{*}\right)^{2} \stackrel{d}{=} R_{d}^{2} \mathcal{B}_{k / 2,1}$ with $\mathcal{B}_{k / 2,1}$ independent of $R_{d}$.

In view of Theorem 12.3.1 of Berman (1992) and Eq. 7.54 the distribution function $F_{k}^{*}$ of $R_{k}^{*}$ is tail equivalent to $F_{k}$, i.e.

$$
\lim _{u \rightarrow \infty} \frac{1-F_{k}(u)}{1-F_{k}^{*}(u)}=1 .
$$

Hence by Lemma 7.3 we obtain

$$
\lim _{n \rightarrow \infty} \frac{\boldsymbol{P}\left\{\boldsymbol{X}>\boldsymbol{t}_{n}\right\}}{\boldsymbol{P}\left\{\boldsymbol{Y}>\boldsymbol{t}_{n}\right\}}=1 .
$$

Let $\boldsymbol{t}_{n}^{*}, n \geq 1$ be the solution of $\mathcal{P}\left(\Sigma^{-1}, \boldsymbol{t}_{n}\right)$. For any $n \geq 1$ we may further write using Eq. 7.59

$$
\begin{aligned}
\boldsymbol{P}\left\{\boldsymbol{Y}>\boldsymbol{t}_{n}\right\} & =\frac{\Gamma(d / 2)}{\pi^{k / 2}|\Sigma|^{1 / 2}} \int_{\boldsymbol{y}>\boldsymbol{t}_{n}}\left[\int_{r \geq\|\boldsymbol{y}\|} r^{-k} d F_{d}(r)\right] d \boldsymbol{y} \\
& =\frac{\Gamma(d / 2) \alpha_{n}^{|J| / 2} \beta_{n}^{-|I|-|J| / 2}}{\pi^{k / 2}|\Sigma|^{1 / 2}} \int_{\boldsymbol{y}>\boldsymbol{v}_{n}\left(\boldsymbol{t}_{n}-\boldsymbol{t}_{n}^{*}\right)}\left[\int_{r \geq\left\|\boldsymbol{t}_{n}^{*}+\boldsymbol{y} / \boldsymbol{v}_{n}\right\|} r^{-k} d F_{d}(r)\right] d \boldsymbol{y} .
\end{aligned}
$$

Proposition 7.1 implies $\boldsymbol{t}_{n, I}^{*}=\boldsymbol{t}_{n, I}$ for all large $n$. Using further Eqs. 7.44 and 3.13 we arrive at

$$
\begin{aligned}
\left\|\boldsymbol{t}_{n}^{*}+\boldsymbol{y} / \boldsymbol{v}_{n}\right\| & =\alpha_{n}\left(1+\frac{2}{\beta_{n} \alpha_{n}}\left[\left(\boldsymbol{t}_{n, I} / \alpha_{n}\right)^{\top} \Sigma_{I I}^{-1} \boldsymbol{y}_{I}+\boldsymbol{y}_{J}^{\top}\left(\Sigma^{-1}\right)_{J J} \boldsymbol{y}_{J} / 2\right](1+o(1))\right)^{1 / 2} \\
& =\alpha_{n}+\delta_{n} q(\boldsymbol{y}) / \beta_{n}, \quad n \rightarrow \infty,
\end{aligned}
$$


with $q(\boldsymbol{y}):=\boldsymbol{t}_{I}^{\top} \Sigma_{I I}^{-1} \boldsymbol{y}_{I}+\boldsymbol{y}_{J}^{\top}\left(\Sigma^{-1}\right)_{J J} \boldsymbol{y}_{J} / 2, \boldsymbol{y} \in \mathbb{R}^{k}$ and $\delta_{n} \in \mathbb{R}$ such that $\lim _{n \rightarrow \infty}$ $\delta_{n}=1$. Proposition 7.1 and Eq. 3.11 imply

$$
\left(\boldsymbol{v}_{n}\left(\boldsymbol{t}_{n}-\boldsymbol{t}_{n}^{*}\right)\right)_{I}=\mathbf{0}_{I}, \quad\left(\boldsymbol{v}_{n}\left(\boldsymbol{t}_{n}-\boldsymbol{t}_{n}^{*}\right)\right)_{J} \leq \mathbf{0}_{J}, \quad n \geq 1,
$$

and

$$
\left(\boldsymbol{v}_{n}\left(\boldsymbol{t}_{n}-\boldsymbol{t}_{n}^{*}\right)\right)_{J}=\left(\frac{\beta_{n}}{\alpha_{n}}\right)^{1 / 2}\left(\boldsymbol{t}_{n, J}-\Sigma_{J I} \Sigma_{I I}^{-1} \boldsymbol{t}_{n, I}\right) \rightarrow \widetilde{\boldsymbol{u}}_{J}, \quad n \rightarrow \infty .
$$

Consequently

$$
\tilde{\boldsymbol{u}}_{J} \leq \mathbf{0}_{J}, \quad \text { and } \lim _{n \rightarrow \infty} \boldsymbol{v}_{n}\left(\boldsymbol{t}_{n}-\boldsymbol{t}_{n}^{*}\right)=\overline{\boldsymbol{u}}
$$

with $\overline{\boldsymbol{u}} \in \mathbb{R}^{k}$ such that $\overline{\boldsymbol{u}}_{I}:=\mathbf{0}_{I}, \overline{\boldsymbol{u}}_{J}:=\widetilde{\boldsymbol{u}}_{J}$. In the following we write for simplicity $\overline{\boldsymbol{u}}(1+o(1))$ instead of $\boldsymbol{v}_{n}\left(\boldsymbol{t}_{n}-\boldsymbol{t}_{n}^{*}\right)$. Define next

$$
H_{n}(x):=\frac{F_{d}\left(\alpha_{n}+x / \beta_{n}\right)-F_{d}\left(\alpha_{n}\right)}{1-F_{d}\left(\alpha_{n}\right)}, \quad n \geq 1, \forall x \geq 0 .
$$

We can write using further Eq. 3.10

$$
\begin{aligned}
& \frac{\boldsymbol{P}\left\{\boldsymbol{Y}>\boldsymbol{t}_{n}\right\}}{1-F_{d}\left(\alpha_{n}\right)} \\
& =\frac{\Gamma(d / 2) \alpha_{n}^{|J| / 2} \beta_{n}^{-|I|-|J| / 2}}{\pi^{k / 2}|\Sigma|^{1 / 2}} \int_{\boldsymbol{y}>\boldsymbol{v}_{n}\left(\boldsymbol{t}_{n}-\boldsymbol{t}_{n}^{*}\right)}\left[\int_{r \geq\left\|\boldsymbol{t}_{n}^{*}+\boldsymbol{y} / \boldsymbol{v}_{n}\right\|} r^{-k} d F_{d}(r) /\left(1-F_{d}\left(\alpha_{n}\right)\right)\right] d \boldsymbol{y} \\
& =\frac{\Gamma(d / 2) \alpha_{n}^{|J| / 2} \beta_{n}^{-|I|-|J| / 2}}{\Gamma((d-k) / 2) \pi^{k / 2}|\Sigma|^{1 / 2}} \int_{\boldsymbol{y}>\overline{\boldsymbol{u}}(1+o(1))}\left[\int_{r \geq \alpha_{n}+\delta_{n} q(\boldsymbol{y}) / \beta_{n}} r^{-k} d F_{d}(r) /\left(1-F_{d}\left(\alpha_{n}\right)\right)\right] d \boldsymbol{y} \\
& =\frac{\Gamma(d / 2) \alpha_{n}^{|J| / 2-k} \beta_{n}^{-|I|-|J| / 2}}{\pi^{k / 2}|\Sigma|^{1 / 2}} I_{n}, \quad n \rightarrow \infty,
\end{aligned}
$$

with

$$
I_{n}:=\int_{\boldsymbol{y}>\overline{\boldsymbol{u}}(1+o(1))}\left[\int_{r \geq \delta_{n} q(\boldsymbol{y})}\left(1+r /\left(\alpha_{n} \beta_{n}\right)\right)^{-k} d H_{n}(r)\right] d \boldsymbol{y} .
$$

By the assumption on the index set $I$ we have

$$
\boldsymbol{t}_{I}^{\top} \Sigma_{I I}^{-1} \boldsymbol{y}_{I}>0, \quad \forall \boldsymbol{y} \in \mathbb{R}^{k}: \boldsymbol{y}_{I}>\mathbf{0}_{I} .
$$

Consequently $q(\boldsymbol{y})>0$ for all $\boldsymbol{y}_{I}>\mathbf{0}_{I}, \boldsymbol{y} \in \mathbb{R}^{k}$. Thus we obtain for all $n$ large

$$
\left(1+r /\left(\alpha_{n} \beta_{n}\right)\right)^{-k} \leq 1, \quad \forall r \in[q(\boldsymbol{y}), \infty),
$$

with $\boldsymbol{y} \in \mathbb{R}^{k}: \boldsymbol{y}_{I}>\mathbf{0}_{I}$. Equation 3.13 implies further

$$
\lim _{n \rightarrow \infty}\left(1+r /\left(\alpha_{n} \beta_{n}\right)\right)^{-k}=1 .
$$


Using Fubini Theorem we have for all $n$ large

$$
\begin{aligned}
I_{n} & =\int_{r \geq 0}\left[\int_{q(\boldsymbol{y}) \delta_{n}<r, \boldsymbol{y}>\overline{\boldsymbol{u}}(1+o(1))}\left(1+r /\left(\alpha_{n} \beta_{n}\right)\right)^{-k} d \boldsymbol{y}\right] d H_{n}(r) \\
& \leq \int_{r \geq 0}\left[\int_{q(\boldsymbol{y}) \delta_{n}<r, \boldsymbol{y}>\overline{\boldsymbol{u}}(1+o(1))} d \boldsymbol{y}\right] d H_{n}(r) .
\end{aligned}
$$

The matrix $\Sigma:=A^{\top} A$ is positive definite since $A$ is non-singular. This implies that $\Sigma_{I I},\left(\Sigma^{-1}\right)_{J J}$ are also positive definite. In view of Eq. 7.56 we can find two positive constants $c_{1}, c_{2}$ such that

$$
\int_{q(\boldsymbol{y})(1+o(1))<r, \boldsymbol{y}_{I}>\mathbf{0}_{I}} d \boldsymbol{y} \leq c_{1} r^{c_{2}}
$$

is valid for all large $n$. Further we have for any $r_{n} \rightarrow r \in(0, \infty)$

$$
\int_{q(\boldsymbol{y}) \delta_{n}<r_{n}, \boldsymbol{y}>\overline{\boldsymbol{u}}(1+o(1))}\left(1+r /\left(\alpha_{n} \beta_{n}\right)\right)^{-k} d \boldsymbol{y} \rightarrow \int_{q(\boldsymbol{y})<r, \boldsymbol{y}>\overline{\boldsymbol{u}}} d \boldsymbol{y}, \quad n \rightarrow \infty .
$$

In view of Lemma 7.5, Eq. 7.54 and the assumption $F \in M D A(\Lambda, w)$ we have

$$
\lim _{n \rightarrow \infty}\left[H_{n}(t)-H_{n}(s)\right]=\exp (-s)-\exp (-t), \quad t>s, t, s \in \mathbb{R},
$$

hence applying Lemma 7.7 we obtain

$$
\begin{aligned}
\lim _{n \rightarrow \infty} I_{n} & =\int_{\boldsymbol{y}>\overline{\boldsymbol{u}}} \int_{r \geq q(\boldsymbol{y})} \exp (-r) d r d \boldsymbol{y} \\
& =\int_{\boldsymbol{y}_{I}>\mathbf{0}_{I}} \exp \left(-\boldsymbol{t}_{I}^{\top} \Sigma_{I I}^{-1} \boldsymbol{y}_{I}\right) d \boldsymbol{y}_{I} \int_{\boldsymbol{y}_{J}>\widetilde{\boldsymbol{u}}_{J}} \exp \left(-\boldsymbol{y}_{J}^{\top}\left(\Sigma^{-1}\right)_{J J} \boldsymbol{y}_{J} / 2\right) d \boldsymbol{y}_{J} \\
& =\frac{(2 \pi)^{|J| / 2}}{\left|\left(\Sigma^{-1}\right)_{J J}\right| \prod_{i \in I} \boldsymbol{t}_{I}^{\top} \Sigma_{I I}^{-1} \boldsymbol{e}_{i}} \boldsymbol{P}\left\{\boldsymbol{Z}_{J}>\widetilde{\boldsymbol{u}}_{J} \mid \boldsymbol{Z}_{I}=\mathbf{0}_{I}\right\},
\end{aligned}
$$

with $\boldsymbol{Z}$ a Gaussian random vector in $\mathbb{R}^{k}$ with covariance matrix $\Sigma$. Clearly Eq. 7.54 implies

$$
\frac{1-F_{d}\left(\alpha_{n}\right)}{1-F_{k}\left(\alpha_{n}\right)}=(1+o(1)) \frac{\alpha_{n} \beta_{n} \Gamma(k / 2)}{2 \Gamma(d / 2)}, \quad n \rightarrow \infty .
$$

The fact that $\Sigma$ is positive definite yields

$$
|\Sigma|=\left|\Sigma_{I I}\right|\left|\left(\left(\Sigma^{-1}\right)_{J J}\right)^{-1}\right|=\frac{\left|\Sigma_{I I}\right|}{\left|\left(\Sigma^{-1}\right)_{J J}\right|}>0
$$

consequently as $n \rightarrow \infty$

$$
\frac{\boldsymbol{P}\left\{\boldsymbol{X}>\boldsymbol{t}_{n}\right\}}{1-F_{k}\left(\alpha_{n}\right)}=(1+o(1)) \frac{\Gamma(k / 2) 2^{k / 2-1} \boldsymbol{P}\left\{\boldsymbol{Z}_{J}>\widetilde{\boldsymbol{u}}_{J} \mid \boldsymbol{Z}_{I}=\mathbf{0}_{I}\right\}}{(2 \pi)^{|I| / 2}\left|\Sigma_{I I}\right|^{1 / 2} \prod_{i \in I} \boldsymbol{t}_{I}^{\top} \Sigma_{I I}^{-1} \boldsymbol{e}_{i}} \alpha_{n}^{1+|J| / 2-k} \beta_{n}^{1-|I|-|J| / 2},
$$

thus the proof follows.

We give next a similar result to Lemma 7.3. 
Lemma 7.8 Let $A, R_{k}, R_{k}^{*}, \boldsymbol{X}, \boldsymbol{Y}$ be as in Lemma 7.3. Assume that the distribution function $F_{k}$ of $R_{k}$ has finite upper endpoint $\omega \in(0, \infty)$ such that $F_{k}(\omega-)=1$ and Eq. 7.45 holds. Let $\boldsymbol{t}, \boldsymbol{t}_{n}, n \in \mathbb{N}$ and I satisfy the assumptions of Theorem 3.3. Then Eq. 7.46 is satisfied.

Proof The assumption on the threshold $\boldsymbol{t}_{n}$ implies for any $\boldsymbol{y}=\left(y_{1}, \ldots, y_{k}\right)^{\top} \in$ $\mathbb{R}^{k} \backslash(-\infty, 0]^{k}, y_{i}>0,1 \leq i \leq k$ such that $\|\boldsymbol{y}\| \leq 1$

$$
\boldsymbol{P}\left\{R_{k} \boldsymbol{y}>\boldsymbol{t}_{n}\right\} \leq \boldsymbol{P}\left\{\left\|R_{k} \boldsymbol{y}\right\| \geq \min _{z \geq \boldsymbol{t}_{n}}\|\boldsymbol{z}\|\right\}=\boldsymbol{P}\left\{R_{k} \geq\left\|\boldsymbol{t}_{n, I}\right\|\right\} \rightarrow \boldsymbol{P}\left\{R_{k} \geq\left\|\boldsymbol{t}_{I}\right\|\right\}=0
$$

as $n \rightarrow \infty$ where we used further the fact that $F_{k}(\omega-)=1$. Hence we have

$$
\lim _{n \rightarrow \infty} \boldsymbol{P}\left\{\boldsymbol{X}>\boldsymbol{t}_{n}\right\}=\lim _{n \rightarrow \infty} \boldsymbol{P}\left\{\boldsymbol{X}^{*}>\boldsymbol{t}_{n}\right\}=0 .
$$

We consider now for simplicity again only the case $\boldsymbol{t}_{n}$ has non-negative components for all $n$ large. Since $R_{k}$ is independent of $\boldsymbol{U}_{k}$ and $R_{k}$ almost surely positive we have

$$
\boldsymbol{P}\left\{\boldsymbol{X}>\boldsymbol{t}_{n}\right\}=\int_{\boldsymbol{y} \in D_{n}} \boldsymbol{P}\left\{R_{k}>\max _{1 \leq i \leq k}\left(t_{n i} / y_{i}\right)\right\} d G(\boldsymbol{y}),
$$

with $G$ the distribution function of $A^{\top} \boldsymbol{U}_{k}$ and $D_{n}:=\left\{\boldsymbol{y} \in \mathbb{R}^{k}: \exists h \in(0, \omega)\right.$ : $\left.h \boldsymbol{y}>\boldsymbol{t}_{n},\|\boldsymbol{y}\|=1, \boldsymbol{y}>\boldsymbol{0}\right\}$. Consequently, for all $\boldsymbol{y},\|\boldsymbol{y}\| \leq 1, y_{i}>0,1 \leq i \leq k$ we have uniformly in $\boldsymbol{y}$

$$
\lim _{n \rightarrow \infty} \frac{\boldsymbol{P}\left\{R_{k}>\max _{1 \leq i \leq k}\left(t_{n i} / y_{i}\right)\right\}}{\boldsymbol{P}\left\{R_{k}^{*}>\max _{1 \leq i \leq k}\left(t_{n i} / y_{i}\right)\right\}}=c
$$

thus

$$
\begin{aligned}
& \int_{\boldsymbol{y}>\mathbf{0},\|\boldsymbol{y}\| \leq 1} \boldsymbol{P}\left\{R_{k}>\max _{1 \leq i \leq k}\left(t_{n i} / y_{i}\right)\right\} d G(\boldsymbol{y}) \\
& =(1+o(1)) c \int_{\boldsymbol{y}>\mathbf{0}} \boldsymbol{P}\left\{R_{k}^{*}>\max _{1 \leq i \leq k}\left(t_{n i} / y_{i}\right)\right\} d G(\boldsymbol{y}) \\
& =(1+o(1)) \boldsymbol{P}\left\{\boldsymbol{Y}>\boldsymbol{t}_{n}\right\}, \quad n \rightarrow \infty,
\end{aligned}
$$

hence the proof is complete.

Proof of Theorem 3.3 First note that Eq. 3.7 implies (see e.g. Leadbetter et al. 1983) $F_{k}(\omega-)=F_{k}(\omega)=1$. The proof is very similar to the proof of Theorem 3.1 using further Lemma 7.4 and Eq. 3.13.

Proof of Theorem 3.4 The asymptotic behaviour of $\boldsymbol{P}\left\{\boldsymbol{X}>\boldsymbol{t}_{n}+\boldsymbol{q}_{n}\right\}$ can be shown utilising the same arguments as in the proof of Theorem 3.1. The proof follows then easily comparing with the asymptotic behaviour of $\boldsymbol{P}\left\{\boldsymbol{X}>\boldsymbol{t}_{n}\right\}$.

Proof of Lemma 3.5 It is well-known (see e.g. Cambanis et al. 1981) that any subvector of $\boldsymbol{X}$ is an elliptical random vector. Equation 4.21 implies $R_{m}^{2} \stackrel{d}{=} R_{k}^{2} \mathcal{B}_{m / 2,(k-m) / 2}$. The proof follows now applying Theorem 12.3.1 in Berman (1992). 
Proof of Theorem 4.1 Let $d>k$ be such that $R_{d} \in \mathcal{M}_{R_{k}}$. Lemma 8.1 implies that the elliptical random vector $\boldsymbol{X}$ possesses a density function $f$ defined by

$$
f(\boldsymbol{x})=\frac{\Gamma(d / 2)}{\Gamma((d-k) / 2) \pi^{k / 2}|\Sigma|^{1 / 2}} \int_{\|\boldsymbol{x}\|}^{\infty}\left(r^{2}-\|\boldsymbol{x}\|^{2}\right)^{(d-k) / 2-1} r^{-(d-2)} d F_{d}(r), \quad \forall \boldsymbol{x} \in \mathbb{R}^{k},
$$

with $F_{d}$ the distribution function of $R_{d}$ and $\Sigma:=A^{\top} A$. Applying Lemma 7. 6 we obtain

$$
f\left(\boldsymbol{t}_{n}\right)=\frac{(1+o(1)) \Gamma(d / 2)}{\pi^{k / 2}|\Sigma|^{1 / 2}}\left(\frac{2 \alpha_{n}}{\beta_{n}}\right)^{(d-k) / 2-1} \alpha_{n}^{-(d-2)}\left[1-F_{d}\left(\alpha_{n}\right)\right], \quad n \rightarrow \infty,
$$

with $\alpha_{n}:=\left\|\boldsymbol{t}_{n}\right\|, \beta_{n}:=w\left(\alpha_{n}\right), n \geq 1$. Equation 4.21 implies $R_{k}^{2} \stackrel{d}{=} R_{d}^{2} \mathcal{B}_{k / 2,(n-k) / 2}$, hence utilising Theorem 12.3.1 of Berman (1992) we obtain

$$
f\left(\boldsymbol{t}_{n}\right)=\frac{(1+o(1)) \Gamma(k / 2)}{2 \pi^{k / 2}|\Sigma|^{1 / 2}} \alpha_{n}^{1-k} \beta_{n}\left[1-F_{k}\left(\alpha_{n}\right)\right], \quad n \rightarrow \infty,
$$

thus the proof is complete.

Proof of Corollary 4.2 Set $\alpha_{n}:=\left\|\boldsymbol{t}_{n, I}^{*}\right\|=\left\|\boldsymbol{t}_{n, I}\right\|, \beta_{n}:=w\left(\alpha_{n}\right), n \geq 1$. In view of Eq. 7.44 (set $\boldsymbol{x}=\mathbf{0}$ therein) we have

$$
\alpha_{n}=\left\|\boldsymbol{t}_{n}^{*}\right\|=\left\|\boldsymbol{t}_{n, I}\right\| \rightarrow \omega, \quad n \rightarrow \infty
$$

thus Eq. 3.13 holds. If $\lim _{n \rightarrow \infty} \boldsymbol{t}_{n, I} / \alpha_{n}=\boldsymbol{t}_{I}$ or $\boldsymbol{y}_{I}=\mathbf{0}_{I}$ we obtain (see above Eq. 7.55)

$$
\left\|\boldsymbol{t}_{n}^{*}+\boldsymbol{y} / \boldsymbol{v}_{n}\right\|=\alpha_{n}+\delta_{n} q(\boldsymbol{y}) / \beta_{n}, \quad n \rightarrow \infty
$$

where $\lim _{n \rightarrow \infty} \delta_{n}=1 . F_{k} \in \operatorname{MDA}(\Lambda, w)$ implies

$$
\lim _{n \rightarrow \infty} \frac{1-F_{k}\left(\left\|\boldsymbol{t}_{n}^{*}+\boldsymbol{y} / \boldsymbol{v}_{n}\right\|\right)}{1-F_{k}\left(\alpha_{n}\right)}=\lim _{n \rightarrow \infty} \frac{1-F_{k}\left(\alpha_{n}+\delta_{n} q(\boldsymbol{y}) / \beta_{n}\right)}{1-F_{k}\left(\alpha_{n}\right)}=\exp (-q(\boldsymbol{y})) .
$$

Further we may write (recall Eq. 3.15)

$$
\lim _{n \rightarrow \infty} \frac{w\left(\left\|\boldsymbol{t}_{n}^{*}+\boldsymbol{y} / \boldsymbol{v}_{n}\right\|\right)}{\beta_{n}}=\lim _{n \rightarrow \infty} \frac{w\left(\alpha_{n}+\delta_{n} q(\boldsymbol{y}) \beta_{n}\right)}{w\left(\alpha_{n}\right)}=1
$$

thus the second claim follows, hence the proof is complete.

Proof of Theorem 5.1 Assume for simplicity that $J$ is not empty. We have by the assumptions (using Proposition 7.1) $h_{i}:=\boldsymbol{t}_{I}^{\top} \Sigma_{I I}^{-1} \boldsymbol{e}_{i}>0, \forall i \in I$ with $\boldsymbol{e}_{i}$ the 
$i$-th unit vector in $\mathbb{R}^{|I|}$. For any $\boldsymbol{x} \in(0, \infty)^{k}$ put $\boldsymbol{q}_{n}:=\boldsymbol{x} / \boldsymbol{v}_{n}, n \geq 1$. Clearly, $\lim _{n \rightarrow \infty} \boldsymbol{v}_{n} \boldsymbol{q}_{n}=\boldsymbol{x}$, hence Eq. 3.20 yields as $n \rightarrow \infty$

$$
\begin{aligned}
\boldsymbol{P}\left\{\boldsymbol{v}_{n} \boldsymbol{X}_{\left[\boldsymbol{t}_{n}\right]}>\boldsymbol{x}\right\} & =\frac{\boldsymbol{P}\left\{\boldsymbol{X}>\boldsymbol{t}_{n}+\boldsymbol{q}_{n}\right\}}{\boldsymbol{P}\left\{\boldsymbol{X}>\boldsymbol{t}_{n}\right\}} \\
& =(1+o(1)) \prod_{i \in I} \exp \left(-h_{i} x_{i}\right) \frac{\boldsymbol{P}\left\{\boldsymbol{Z}_{J}>\widetilde{\boldsymbol{u}}_{J}+\boldsymbol{x}_{J} \mid \boldsymbol{Z}_{I}=\mathbf{0}_{I}\right\}}{\boldsymbol{P}\left\{\boldsymbol{Z}_{J}>\widetilde{\boldsymbol{u}}_{J} \mid \boldsymbol{Z}_{I}=\mathbf{0}_{I}\right\}} .
\end{aligned}
$$

The convergence of $\boldsymbol{P}\left\{\boldsymbol{v}_{n}\left(\boldsymbol{X}_{\left[\boldsymbol{t}_{n}\right]}\right)_{L}>\boldsymbol{x}_{L}\right\}, \boldsymbol{x}_{L} \in \mathbb{R}^{|L|}$ with $L$ a non-empty index set follows with the same arguments.

Proof of Corollary 5.2 The proof follows by Theorem 5.1 since $\widetilde{\boldsymbol{u}}_{J}=\mathbf{0}_{J}$.

Proof of Corollary 5.3 Let $h_{i}>0, \forall i \in I$ be as in Theorem 5.1, and assume again for simplicity that $J$ is not empty. For any $n \geq 1$ and $\boldsymbol{x} \in \mathbb{R}^{k}$ with $\boldsymbol{x}_{I}>\boldsymbol{0}_{I}$ we have $\left(\boldsymbol{x} / \boldsymbol{v}_{n}+\boldsymbol{t}_{n}\right)_{I} \geq \boldsymbol{t}_{n, I}, n \geq 1$, hence we may write

$$
\boldsymbol{P}\left\{\boldsymbol{v}_{n} \boldsymbol{X}_{\left[\boldsymbol{t}_{n} ; I\right]}>\boldsymbol{x}\right\}=\frac{\boldsymbol{P}\left\{\boldsymbol{X}>\boldsymbol{x} / \boldsymbol{v}_{n}+\boldsymbol{t}_{n}\right\}}{\boldsymbol{P}\left\{\boldsymbol{X}_{I}>\boldsymbol{t}_{n, I}\right\}} .
$$

In view of the above results and Eq. 3.20 we obtain

$$
\lim _{n \rightarrow \infty} \boldsymbol{P}\left\{\boldsymbol{v}_{n} \boldsymbol{X}_{\left[\boldsymbol{t}_{n} ; I\right]}>\boldsymbol{x}\right\}=\boldsymbol{P}\left\{\boldsymbol{Z}_{J}>\widetilde{\boldsymbol{u}}_{J}+\boldsymbol{x}_{J} \mid \boldsymbol{Z}_{I}=\mathbf{0}_{I}\right\}
$$

Convergence of $\boldsymbol{P}\left\{\boldsymbol{v}_{n}\left(\boldsymbol{X}_{\left[\boldsymbol{t}_{n} ; I\right]}\right)_{L}>\boldsymbol{x}_{L}\right\}, \boldsymbol{x}_{L} \in \mathbb{R}^{|L|}$ with $L$ a non-empty index set follows with the same arguments, thus the proof.

Proof of Corollary 5.5 In view of Lemma 3.5 the distribution function $G$ of $X_{1}$ is in the Gumbel max-domain of attraction with the scaling function $w$. Further, for $K \subset\{1, \ldots, k\}$ we have that $\boldsymbol{X}_{K}$ is an elliptical random vector with a density function $f_{K}$. In particular the distribution function $G$ possesses a density function $G^{\prime}$. The proof follows now easily using Theorem 4.1 and Theorem 5.4.

Proof of Theorem 5.4 By the assumptions $\boldsymbol{X}$ possesses the density function $f$ given in Eq. 4.22. Lemma 8.1 implies that $\boldsymbol{X}_{I}$ possesses the density function $f_{I}$ given by

$$
\begin{aligned}
f_{I}\left(\boldsymbol{x}_{I}\right) & =\frac{\Gamma(k / 2)}{\Gamma((k-m) / 2) \pi^{m / 2}\left|\Sigma_{I I}\right|^{1 / 2}} \int_{\left\|\boldsymbol{x}_{I}\right\|}^{\infty}\left(r^{2}-\left\|\boldsymbol{x}_{I}\right\|^{2}\right)^{(k-m) / 2-1} r^{-(k-2)} d F_{k}(r), \\
\boldsymbol{x} & \in \mathbb{R}^{k}
\end{aligned}
$$

with $\Sigma:=A^{\top} A, m:=|I|$. Hence $\boldsymbol{V}_{n, J}, n \geq 1$ possesses the density function $h_{n, J}$ defined by

$$
h_{n, J}\left(\boldsymbol{x}_{J}\right)=\left(\frac{\alpha_{n}}{\beta_{n}}\right)^{(k-m) / 2} \frac{f\left(\boldsymbol{x}_{n}\right)}{f_{I}\left(\boldsymbol{t}_{n, I}\right)}
$$


with

$$
\boldsymbol{x}_{n} \in \mathbb{R}^{k}: \quad \boldsymbol{x}_{n, I}:=\boldsymbol{t}_{n, I}, \quad \boldsymbol{x}_{n, J}=\left(\beta_{n} / \alpha_{n}\right)^{1 / 2} \boldsymbol{x}_{J}+\Sigma_{J I} \Sigma_{I I}^{-1} \boldsymbol{t}_{n, I}, \quad n \geq 1 .
$$

In view of Eq. 7.57

$$
\begin{aligned}
h_{n, J}\left(\boldsymbol{x}_{J}\right)= & \left(\frac{\alpha_{n}}{\beta_{n}}\right)^{(k-m) / 2} \frac{\Gamma(d / 2) \Gamma((k-m) / 2)}{\Gamma((d-k) / 2) \Gamma(k / 2) \pi^{(k-m) / 2}\left|\left(\Sigma^{-1}\right)_{J J}\right|^{1 / 2}} \\
& \times \frac{\int_{\left\|\boldsymbol{x}_{n}\right\|}^{\infty}\left(r^{2}-\left\|\boldsymbol{x}_{n}\right\|^{2}\right)^{(d-k) / 2-1} r^{-(d-2)} d F_{d}(r)}{\int_{\alpha_{n}}^{\infty}\left(r^{2}-\alpha_{n}^{2}\right)^{(k-m) / 2-1} r^{-(k-2)} d F_{k}(r)}
\end{aligned}
$$

with $F_{d}$ the distribution function of $R_{d} \in \mathcal{M}_{R_{k}}$. Applying Theorem 12.3.1 in Berman (1992) (see Theorem 7.2 in Appendix) we have $F_{k} \in M D A(\Lambda, w)$, hence Eq. 4.24 implies

$$
\lim _{n \rightarrow \infty} \frac{f_{I}\left(\boldsymbol{t}_{n, I}\right)}{\alpha_{n}^{1-m} \beta_{n}\left[1-F_{m}\left(\alpha_{n}\right)\right]}=\frac{(1+o(1)) \Gamma(m / 2)}{2 \pi^{m / 2}\left|\Sigma_{I I}\right|^{1 / 2}} .
$$

In view of Eq. 4.26

$$
\frac{f\left(\boldsymbol{x}_{n}\right)}{\alpha_{n}^{1-k} \beta_{n}\left[1-F_{k}\left(\alpha_{n}\right)\right]} \rightarrow \frac{(1+o(1)) \Gamma(k / 2)}{2 \pi^{k / 2}|\Sigma|^{1 / 2}} \exp \left(-\boldsymbol{x}_{J}^{\top}\left(\Sigma^{-1}\right)_{J J} \boldsymbol{x}_{J} / 2\right), \quad n \rightarrow \infty
$$

holds locally uniformly for $\boldsymbol{x}_{I} \in \mathbb{R}^{m}$. Equation 4.21 yields $R_{m}^{2} \stackrel{d}{=} R_{k}^{2} \mathcal{B}_{m / 2,(k-m) / 2}$. In the light of Theorem 12.3.1 in Berman (1992) we have

$$
\frac{1-F_{m}\left(\alpha_{n}\right)}{1-F_{k}\left(\alpha_{n}\right)}=(1+o(1))\left(\alpha_{n} \beta_{n}\right)^{-(k-m) / 2} 2^{(k-m) / 2} \frac{\Gamma(k / 2)}{\Gamma(m / 2)}, \quad n \rightarrow \infty .
$$

Using further Eq. 7.57 we have that

$$
h_{n, J}\left(\boldsymbol{x}_{J}\right) \rightarrow \frac{\exp \left(-\boldsymbol{x}_{J}^{\top}\left(\Sigma^{-1}\right)_{J J} \boldsymbol{x}_{J} / 2\right)}{(2 \pi)^{(k-m) / 2}\left|\left(\Sigma^{-1}\right)_{J J}\right|^{1 / 2}}=\varphi\left(\boldsymbol{x}_{J}\right), \quad n \rightarrow \infty
$$

holds locally uniformly in $\mathbb{R}^{k-m}$ with $\varphi$ the distribution function of $\boldsymbol{Z}_{J} \mid \boldsymbol{Z}_{I}=\mathbf{0}_{I}$ and $\boldsymbol{Z}$ a standard Gaussian random vector in $\mathbb{R}^{k}$ with covariance matrix $\Sigma$.

Proof of Theorem 5.6 Let $G$ denote the distribution function of $S_{1}$. For $n>1$ set in the following

$$
b_{n}:=G^{-1}(1-1 / n), \quad A_{n}:=\sqrt{1-\rho^{2}}\left(b_{n} / w\left(b_{n}\right)\right)^{1 / 2}, \quad B_{n}=\rho b_{n} .
$$

In view of Theorem 12.3.1 in Berman (1992) the distribution function $G$ is in the max-domain of attraction of $\Lambda$ with the scaling function $w$. Hence we have

$$
w\left(b_{n}\right)\left(X_{n: n}-b_{n}\right) \stackrel{d}{\rightarrow} U, \quad n \rightarrow \infty,
$$


where $U \sim \Lambda$. Corollary 5.2 implies further that

$$
\lim _{n \rightarrow \infty} \boldsymbol{P}\left\{Y_{1} \leq A_{n} y+B_{n} \mid X_{1}>x / w\left(b_{n}\right)+b_{n}\right\}=\boldsymbol{P}\{Z \leq y\}
$$

holds uniformly for $x, y$ in compact subsets of $I R$ with $Z$ a standard Gaussian random variable. Consequently utilising Eq. 5.35 we obtain

$$
\frac{Y_{[n: n]}-B_{n}}{A_{n}} \stackrel{d}{\rightarrow} Z, \quad n \rightarrow \infty .
$$

The joint convergence follows easily, hence the proof is complete.

\section{Appendix}

We state for ease of reference two results which are crucial for the proofs above.

Lemma 8.1 Let $\boldsymbol{X} \stackrel{d}{=} R A^{\top} \boldsymbol{U}_{k}$ be an elliptical random vector in $\mathbb{R}^{k}, k \geq 2$, with $R$ an almost surely positive random radius independent of $\boldsymbol{U}_{k}$, and $\bar{A} \in \mathbb{R}^{k \times k}$ a non-singular matrix. Let $I \subset\{1, \ldots, k\}$ be non-empty index set with $m<k$ elements. Then we have for any $\boldsymbol{x} \in \mathbb{I}^{k}$

$$
\begin{aligned}
\boldsymbol{P}\left\{\boldsymbol{X}_{I}>\boldsymbol{x}_{I}\right\}= & \frac{\Gamma(k / 2)}{\Gamma((k-m) / 2) \pi^{m / 2}|\Sigma|^{1 / 2}} \\
& \times \int_{\boldsymbol{y}_{I}>\boldsymbol{x}_{I}}\left[\int_{\left\|\boldsymbol{y}_{I}\right\|}^{\infty}\left(r^{2}-\left\|\boldsymbol{y}_{I}\right\|^{2}\right)^{(k-m) / 2-1} r^{-(k-2)} d F(r)\right] d \boldsymbol{y}_{I},
\end{aligned}
$$

with $F$ the distribution function of $R$ and $\Sigma:=A^{\top} A$.

The above result can be found in Cambanis et al. (1981); Fang et al. (1990) or Anderson and Fang (1990).

Theorem 8.2 [Theorem 12.3.1 of Berman (1992)] Let $H$ be an univariate distribution function with upper endpoint $\omega \in(0, \infty]$, and let $Y \sim \mathcal{B}_{a, b}$ be a Beta distributed random variable with positive parameters $a$ and $b$. If $H$ is in the maxdomain of attraction of $\Lambda$ with the scaling function $w$, then we have

$$
\boldsymbol{E}\left\{1-H\left(u(1-Y)^{-1 / 2}\right)\right\}=(1+o(1))\left(\frac{2}{u w(u)}\right)^{a} \frac{\Gamma(a+b)}{\Gamma(b)}[1-H(u)], \quad u \uparrow \omega .
$$

Acknowledgements I would like to thank the Referee of the paper for some suggestions and Dr. Marco Collenberg for several fruitful discussions. 


\section{References}

Abdous, B., Fougères, A.-L., Ghoudi, K.: Extreme behaviour for bivariate elliptical distributions. Can. J. Stat. 33(3), 317-334 (2005)

Abdous, B., Fougères, A.-L., Ghoudi, K., Soulier, P.: Estimation of bivariate excess probabilities for elliptical models. (2006) http://www.arXiv:math.ST/0611914

Anderson, T.W., Fang, K.T.: On the theory of multivariate elliptically contoured distributions and their applications. In: Fang, K.T., Anderson, T.W. (eds.) Statistical Inference in Elliptically Contoured and Related Distributions, pp.1-23. Allerton Press, New York (1990)

Berman, M.S.: Sojourns and extremes of stationary processes. Ann. Probab. 10, 1-46 (1982)

Berman, M.S.: Sojourns and extremes of Fourier sums and series with random coefficients. Stoch. Proc. Appl. 15, 213-238 (1983)

Berman, M.S.: Sojourns and Extremes of Stochastic Processes. Wadsworth \& Brooks Cole, Boston (1992)

Butler, A., Tawn, J.A.: Conditional extremes of a markov chain. (2007) (in press)

Cambanis, S., Huang, S., Simons, G.: On the theory of elliptically contoured distributions. J. Multivariate Anal. 11, 368-385 (1981)

Dai, M., Mukherjea, A.: Identification of the parameters of a multivariate normal vector by the distribution of the minimum. J. Theoretical Prob. 14(1), 267-298 (2001)

De Haan, L.: On Regular Variation and its Applications to the Weak Convergence of Sample Extremes. Mathematisch Centrum Amsterdam, Netherlands (1970)

De Haan, L., Ferreira, A.: Extreme Value Theory. An Introduction. Springer, Berlin (2006)

Eddy, W.F., Gale, J.D.: The convex hull of a spherically symmetric sample. Adv. Appl. Probab. 13, 751-763 (1981)

Falk, M., Hüsler, J., Reiss R.-D.: Laws of small numbers: extremes and rare events, vol. 23, 2nd edn. DMV Seminar, Birkhäuser, Basel (2004)

Fang, K.-T., Kotz, S., Ng, K.-W.: Symmetric Multivariate and Related Distributions. Chapman \& Hall, London (1990)

Fang, K., Zhang, Y.: Generalized Multivariate Analysis. Springer, Berlin (1990)

Galambos, J.: Asymptotic Theory of Extreme Order Statistics, 2nd edn. Krieger, Malabar, Florida (1987)

Gale, J.D.: The Asymptotic Distribution of the Convex Hull of a Random Sample. Ph.D. thesis, Carnegie-Mellon University (1980)

Gupta, A.K., Varga, T.: Elliptically Contoured Models in Statistics. Kluwer, Dordrecht (1993)

Hashorva, E.: Asymptotics of the dominated Gaussian maxima. Extremes 5, 353-368 (2003)

Hashorva, E.: Asymptotics and bounds for multivariate Gaussian tails. J. Theoretical Prob. 18(1) 79-97 (2005a)

Hashorva, E.: Extremes of asymptotically spherical and elliptical random vectors. Insur. Math. Econ. 36(3), 285-302 (2005b)

Hashorva, E.: Elliptical triangular arrays in the max-domain of attraction of Hüsler-Reiss distribution. Statist. Probab. Lett. 72(2), 125-135 (2005c)

Hashorva, E.: Gaussian approximation of conditional elliptical random vectors. Stoch Model 22, 441-457 (2006a)

Hashorva, E.: On the multivariate Hüsler-Reiss distribution attracting the maxima of elliptical triangular arrays. Stat. Prob. Letters 76, 2027-2035 (2006b)

Hashorva, E.: A novel class of bivariate max-stable distributions. Stat. Prob. Letters, 76(10) 1047-1055 (2006c)

Hashorva, E.: Extremes and asymptotic dependence of elliptical random vectors. In: Ahsanulah, M., Kirmani, S. (eds.) Extreme Value Distributions, pp. 159-179. Nova Science, New York (2007a)

Hashorva, E.: Conditional limiting distribution of type III elliptical random vectors. J. Multivariate Anal. 98, 282-194 (2007b)

Hashorva, E.: Exact tail asymptotics for Type I bivariate elliptical distributions. Albanian Journal of Mathematics 1(2), 99-114 (2007c)

Hashorva, E., Hüsler, J.: On asymptotics of multivariate integrals with applications to records. Stoc. Models 18(1), 41-69 (2002a)

Hashorva, E., Hüsler, J.: Remarks on compound poisson approximation of gaussian random sequences. Statist. Prob. Letters 57, 1-8 (2002b) 
Hashorva, E., Hüsler J.: On multivariate Gaussian tails. Ann. Inst. Statist. Math. 55(3), 507-522 (2003)

Heffernan, J.E., Tawn, J.A.: A conditional approach for multivariate extreme values. J. R. Stat. Soc. Ser. B Stat. Methodol. 66(3), 497-546 (2004)

Heffernan, J.E., Resnick, S.I.: Limit laws for random vectors with an extreme component. (2005) (http://www.maths.lancs.ac.uk/ currie/Papers/ConditModel.pdf)

Hüsler, J., Reiss, R-D.: Maxima of normal random vectors: between independence and complete dependence. Statist. Probab. Lett. 7, 283-286 (1989)

Joshi, S.N., Nagaraja, H.N.: Joint distribution of maxima of concomitants of subsets of order statistics. Bernoulli 1, 245-255 (1995)

Kano, Y.: Consistency property of elliptical probability density functions. J. Multivariate Anal. 51, 139-147 (1994)

Kaufmann, E., Reiss, R.-D.: On conditional distributions of nearest neighbors. J. Multivariate Anal. 42(1), 67-76 (1992)

Klüppelberg, C., Kuhn, K., Peng, L.: Estimating the tail dependence of an elliptical distribution. Bernoulli 13(1), 229-251 (2007)

Kotz, S.: Multivariate distributions at a cross-road. In: Patil, G.P., Kotz, S., Ord, J.K. (eds.) Statistical Distributions in Scientific Work, 1, pp. 247-240. Riedel, Dortrecht (1975)

Kotz, S., Ostrovskii, I.V.: Characteristic functions of a class of elliptical distributions. J. Multivariate Analysis. 49(1), 164-178 (1994)

Kotz, S., Nadarajah, S.: Extreme Value Distributions, Theory and Applications. Imperial College Press, London (2000)

Leadbetter, M.R., Lindgren, G., Rootzén, H.: Extremes and related properties of random sequences and processes. Springer, New York (1983)

Ledford, W.A., Twan, A.J.: On the tail concomitant behaviour for extremes. Adv. Appl. Prob. 30, 197-215 (1998)

Nagaraja, H.N., David, H.A.: Distribution of the maximum of concomitants of selected order statistics. Ann. Statist. 1, 478-494 (1994)

Reiss, R-D.: Approximate Distributions of Order Statistics: With Applications to Nonparametric Statistics. Springer, New York (1989)

Resnick, S.I.: Extreme Values, Regular Variation and Point Processes. Springer, New York (1987)

Szabłowski, P.L.: Expansions of $E(X \mid Y+\epsilon X)$ and their applications to the analysis of elliptically contoured measures. Comput. Math. Appl. 19(5), 75-83 (1990) 